\title{
Unsupervised Methods for Detection of Neural States: Case Study of Hippocampal-Amygdala Interactions
}

\author{
DFrancesco Cocina, Andreas Vitalis, and Amedeo Caflisch
}

https://doi.org/10.1523/ENEURO.0484-20.2021

Biochemistry department, University of Zurich, Zurich, Switzerland $\mathrm{CH}-8057$

\begin{abstract}
The hippocampus and amygdala are functionally coupled brain regions that play a crucial role in processes involving memory and learning. Because interareal communication has been reported both during specific sleep stages and in awake, behaving animals, these brain regions can serve as an archetype to establish that measuring functional interactions is important for comprehending neural systems. To this end, we analyze here a public dataset of local field potentials (LFPs) recorded in rats simultaneously from the hippocampus and amygdala during different behaviors. Employing a specific, time-lagged embedding technique, named topological causality (TC), we infer directed interactions between the LFP band powers of the two regions across six frequency bands in a time-resolved manner. The combined power and interaction signals are processed with our own unsupervised tools developed originally for the analysis of molecular dynamics simulations to effectively visualize and identify putative, neural states that are visited by the animals repeatedly. Our proposed methodology minimizes impositions onto the data, such as isolating specific epochs, or averaging across externally annotated behavioral stages, and succeeds in separating internal states by external labels such as sleep or stimulus events. We show that this works better for two of the three rats we analyzed, and highlight the need to acknowledge individuality in analyses of this type. Importantly, we demonstrate that the quantification of functional interactions is a significant factor in discriminating these external labels, and we suggest our methodology as a general tool for large, multisite recordings.
\end{abstract}

Key words: amygdala; hippocampus; interactions; local field potential; neural states; unsupervised methods

\section{Significance Statement}

We develop an analysis pipeline for neuroscience datasets. We test it on a published example of multielectrode recordings of rats in a range of behaviors: running on a track, sleeping, collecting rewards, etc. We adopt nonlinear analysis techniques that are able to quantify directed interactions between different signals, here oscillations of two brain regions in different frequency bands. Using the entire recordings and, importantly, distinguishing each animal, we provide a high-resolution overview of the functional interplay of the two regions. Putative neural states that the animals can be in are derived from a time-aware clustering of the large datasets. When discriminating experimental annotations like run speed, we provide evidence that our methodology outperforms common clustering techniques.

Received November 13, 2020; accepted September 14, 2021; First published September 20, 2021.

The authors declare no competing financial interests.

Author contributions: F.C., A.V., and A.C. designed research; F.C. performed research; F.C. analyzed data; F.C., A.V., and A.C. wrote the paper.

This work was supported financially by an excellence grant of the Swiss National Science Foundation (31003A_169007) to A.C.

Acknowledgements: We thank Gabrielle Girardeau and György Buzsáki for publicly sharing their data, Gabrielle Girardeau for helpful comments and Davide Garolini for many useful discussions and for the development of the R package 'CampaRi'.

Correspondence should be addressed to Andreas Vitalis at a.vitalis@ bioc.uzh.ch or Amedeo Caflisch at caflisch@bioc.uzh.ch.

https://doi.org/10.1523/ENEURO.0484-20.2021

Copyright (C) 2021 Cocina et al.

This is an open-access article distributed under the terms of the Creative Commons Attribution 4.0 International license, which permits unrestricted use, distribution and reproduction in any medium provided that the original work is properly attributed. 


\section{Introduction}

A variety of oscillation-related phenomena have been identified within the hippocampus and amygdala, and they are frequently associated with various learning and memory processes that strongly rely on these two brain structures (Paré et al., 2002; Buzsáki and Draguhn, 2004; Colgin, 2016). The coordination between the local field potentials (LFPs) of hippocampus and amygdala, in particular the basolateral complex [basolateral amygdala (BLA)], was highlighted and shown to involve different frequency ranges and interaction modalities (Bocchio et al., 2017; Pesaran et al., 2018). Various studies investigated a number of stimuli and behavioral aspects related to emotional processing, in particular connected to fear and safety, both in rodents (Seidenbecher et al., 2003; Popa et al., 2010; Likhtik et al., 2014; Stujenske et al., 2014) as well as in epileptic patients (Zheng et al., 2017, 2019; Kirkby et al., 2018). These analyses often focus on selected time periods, such as rapid eye movement (REM) sleep or behavioral stages in experiments relying on conditioned fear, and they investigate specific questions of memory-related processes, such as consolidation or retrieval, in specific frequency bands (Bocchio et al., 2017).

All of these results suggest, as a whole, persistent yet diverse ways in which hippocampus and BLA interact with each other, and this set of coupled regions can be extended to other parts of the brain, such as prefrontal or entorhinal cortex (Bauer et al., 2007; Stujenske et al., 2014). Clearly, these interactions might extend beyond the specific cases observed so far in the literature. Indeed, more recent studies shed light on additional functions of both hippocampus and amygdala (Sawangjit et al., 2018; Gründemann et al., 2019; Schapiro et al., 2019), thus broadening the scope for the functions of interareal communication patterns. To arrive at and/or test new hypotheses, it would thus be helpful to collect a global picture of this communication flow where no restrictions to specific time frames or features are imposed, as well as to explore new workflows to achieve this goal in an efficient manner. The potential for new discoveries can, in principle, be maximized if all the information in the time series is preserved. This stipulates that averaging operations, for example, across time windows, sessions, or animals, should be avoided as they may mask variance of interest.

In this work we propose a data-driven procedure to explore an extensive dataset of recordings of LFPs and unveil potential neural states in the context of interactions between regions. The unsupervised procedure is applied here in the well-studied hippocampus-amygdala system, but it can be applied equally well to other areas of the brain. We use a dataset of simultaneous recordings in the rat hippocampus and BLA (Girardeau et al., 2017a), publicly available on https://crcns.org/ (Girardeau et al., 2017b), which comprises multiple behavioral epochs. In these experiments, rats run on a linear track with water rewards placed at the track's ends. A negative (aversive) stimulus in the form of an air puff is delivered in the run epoch and only when the animal travels along one of the two directions (Fig. 1A). During the non-REM (NREM) sleep phases following each run epoch, reactivations of joint neural activity of the hippocampus and the BLA were found to be modulated by hippocampal sharp-wave ripples (SWRs). Moreover, these reactivations were more pronounced for signals corresponding to the direction where the rat faced the aversive stimulus (Girardeau et al., 2017a). The aforementioned results corroborate the hypothesis that contextual, emotional memories derive from the integration of hippocampal neural activity, which encodes the spatial information, with activity in the amygdala, which assigns an emotional value to specific events (Phillips and LeDoux, 1992; Zelikowsky et al., 2014; Beyeler et al., 2016; Kim and Payne, 2020), and shed light on new mechanisms through which the two brain regions influence each other.

We conducted a multistage exploratory analysis of directed interactions between hippocampus and BLA and of neural states associated with both activity and functional connectivity patterns. The analysis focuses on the mutual influence between LFP band powers in six different frequency ranges across the two brain regions, and it can be partitioned into three major steps. First, a standard comparison of average interaction values is conducted between relevant behavioral and physiological epochs. Among the different interaction patterns, $\theta$ oscillations and SWRs in particular carry strong signatures that distinguish different epochs. Second, the information provided by the interactions and LFP power is visualized at maximal time resolution on the global dataset, i.e., without restricting the search to specific epochs or features. We highlight four distinct patterns in these features that are qualitatively identifiable across three different rats. Third, an identification of distinct clusters, or states, in the hippocampus-BLA system is performed in an unsupervised way. More precisely, each state corresponds to a specific pattern that combines both the internal activity within each anatomical area, represented by the LFPs, and the ongoing reciprocal interactions between the two regions. We show that the contribution of interactions plays a significant role for the quality with which such states explain the, for example, behavioral labels that the experiments are annotated with.

The full description of the dataset and techniques is found in Materials and Methods, and the outcomes of the three-stage procedure are presented in Results. For ease of reading, the Results section also includes conceptual explanations of our methodology, both in pictorial and in text form. The analysis yields both expected and unexpected insights, and in the Discussion section we provide an appraisal of these insights both in terms of our chosen methodology and in terms of the biology of the hippocampus-amygdala system.

\section{Materials and Methods}

\section{Experimental design and behavior}

A full description of the experiment is given in the original article (Girardeau et al., 2017a). Here, we merely recapitulate the salient aspects that concern our analysis. Four male Long-Evans rats were trained to run back and forth on a linear track to obtain water rewards placed at 
a

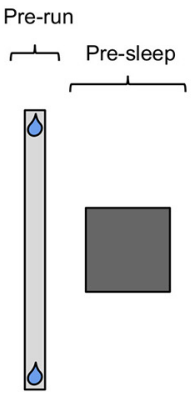

Run

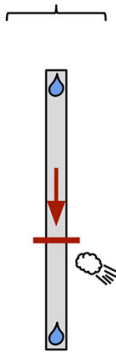

b

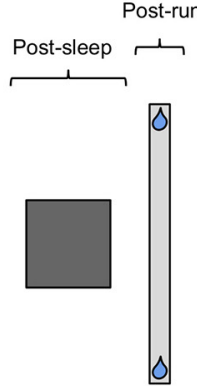

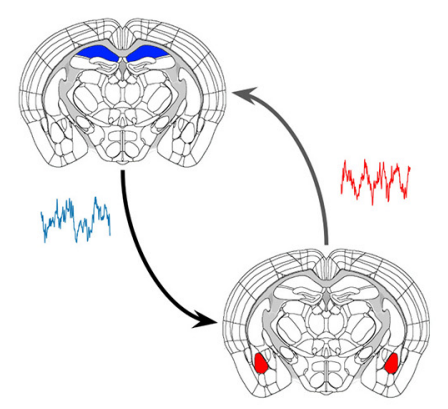

C

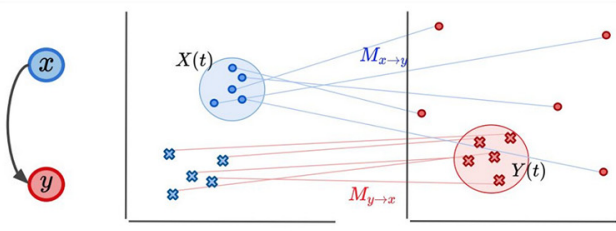

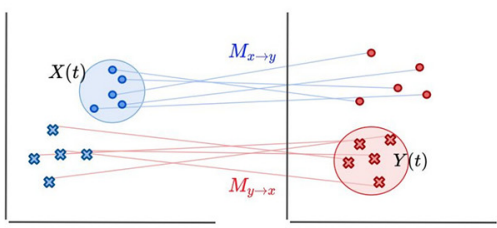

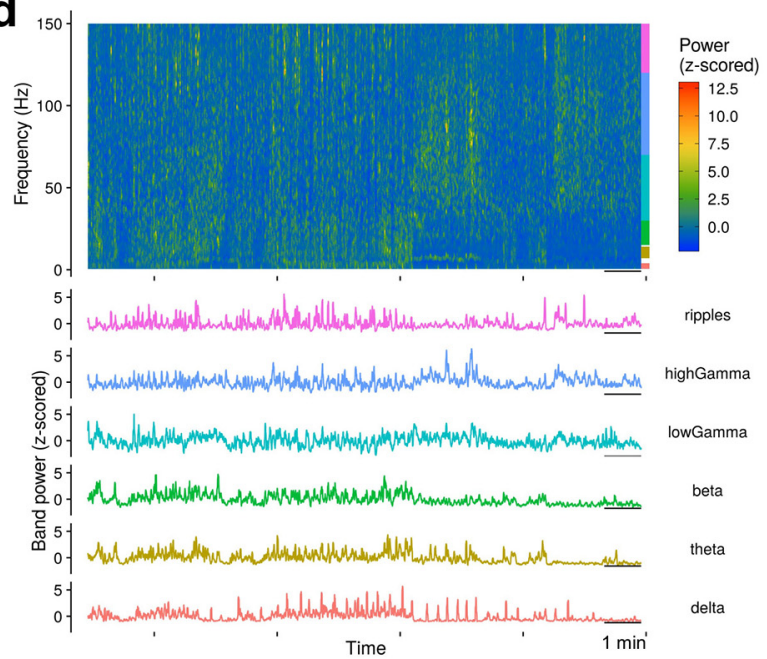

e

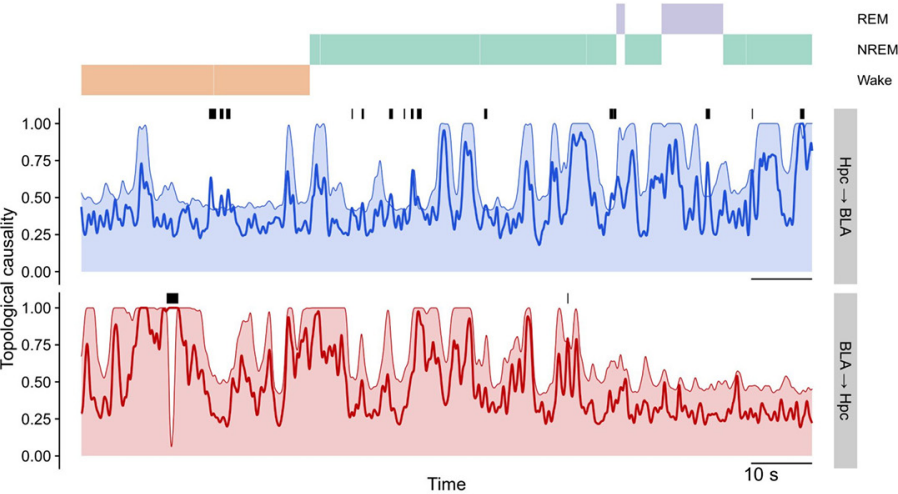

Figure 1. Experiment, hippocampus-BLA interactions, and TC. A, Description of the experiment. In the pre-run epoch (left), the rat runs back and forth freely on the linear track, receiving water rewards at both ends ( $\sim 3$ min each). Successively, the animal is located in a separate environment for sleeping ( $\sim 2-3 \mathrm{~h})$. In the run epoch (middle), back on the linear track, the rat experiences an air puff in a fixed location but only when moving in one specific direction ( $\sim 30 \mathrm{~min})$. This direction and the precise location of the air puff are set randomly. The session is terminated by post-sleep and post-run epochs (right), which have the same modalities of the pre-sleep and pre-run epochs, respectively. $\boldsymbol{B}$, LFPs are recorded in the dorsal hippocampus and in the BLA. Only LFP oscillations from the right BLA are used. $\boldsymbol{C}$, Illustration of the TC method. Two different scenarios of interaction are shown. In the upper panels, $x$ driving $y$ unilaterally implies that the mapping $M_{y \rightarrow x}$ preserves the local neighborhood of $Y$ (the embedded space of $y$ ) when projected onto $X$ (the embedded space of $x$ ). Conversely, when a neighborhood in $X$ is projected onto $Y$, the points are scattered over the whole dynamical space. This is because $x$ evolves independently of the dynamics of $y$, which implies that $M_{x \rightarrow y}$ is ill-defined (its Jacobian diverges everywhere). Instead, when the interactions are bidirectional (lower panel), $X$ contains information about $Y$, so that $M_{x \rightarrow y}$ is well-defined and preserves the local neighborhood of $X$ when mapped to $Y$. $\boldsymbol{D}$, Spectogram of 15-min recordings in the hippocampus (top) along with the six different band powers extracted from it (bottom). Frequency ranges are indicated by colored bars on the side of the spectrogram (ripples band extends up to $250 \mathrm{~Hz}$ ). Power densities in the spectrogram are z-scored within each frequency bin. The band powers (bottom panel) are used to calculate the TC values. $\boldsymbol{E}$, An example of a TC time series of $\sim 90 \mathrm{~s}$ across different sleep phases (color labels on top) in both directions of putative influence. The actual values (solid lines) exceed the chance level (shaded areas, 95\% quantile from shuffling test, see Materials and Methods, Topological causality) only for short, sporadic intervals (black bars on top of the time series). In the final TC time series, non-significant values are zeroed explicitly. The full description of the TC method is provided in Materials and Methods, Topological causality. The alignment of the embeddings of the two time series of band powers is described in Materials and Methods, Alignment settings, and shown in Extended Data Figure 1-1.

both ends. Three silicon probes were implanted above the amygdala, left and right, and in the dorsal CA1 hippocampal region. The probes in the hippocampus had four shanks with 32 recording channels in total (NeuroNexus H32, A-style, Buzsaki32 design). The amygdala electrodes featured eight shanks with 64 channels in total (NeuroNexus H64, A-style, Buzsaki64 design) and were lowered by $140 \mu \mathrm{m}$ every day at the end of each session. Only LFPs from the right BLA were used in the analysis; the number of selected channels recording in this region varied from a minimum of 24 to a maximum of 56. Recording sessions that exhibited low numbers of channels in the BLA, indicated signal degradation, or featured too few aversive stimuli (air puff events) were discarded. Eventually, we kept seven sessions for Rat1 and Rat2, and six for Rat3. The rats analyzed here coincide with the three animals, out of the original four, used in the reactivation analysis of Girardeau and colleagues. Specifically, Rat1, Rat2, and Rat3 here correspond, respectively, to Rat1, Rat3, and Rat4 of the published work (Girardeau et 
al., 2017a) as well as to Rat08, Rat10, and Rat11 in the online dataset (Girardeau et al., 2017b).

The position of the rat was tracked by the two LEDs attached to the head of the animal. Speed was computed as the average value between the two velocities but only when both LEDs were active. Safe trajectory periods were identified as those intervals between consecutive reward events, on opposite ends of the track, when no air puff was recorded. Conversely, we identified as "aversive" trajectories those intervals between a reward and an air puff event, or between multiple air puffs. The identification of different sleep phases (NREM/REM) was done with the help of a k-means clustering of the ratios of the $\delta$ to $\theta$ powers in the hippocampus (higher ratios point to NREM and vice versa), which we extracted from the spectrograms, see LFP preprocessing. The identification of NREM/REM was performed independently for each of the channels, and the final annotation was chosen as the consensus across channels. These data were restricted to periods of immobility, i.e., a sustained speed of below $3 \mathrm{~cm} / \mathrm{s}$ for at least $30 \mathrm{~s}$, with only brief $(<0.5 \mathrm{~s})$ stretches of exceeding this threshold being tolerated (Maingret et al., 2016; QuietPeriods.m and Brainstates.m in MATLAB toolbox FMAtoolbox). Results from spike sorting, units of classification (pyramidal/interneuron), and the SWR timestamps are provided in the original dataset. The latter were identified by the authors by filtering the hippocampal LFP between 100 and $200 \mathrm{~Hz}$, then squaring and normalizing it (z-score; Girardeau et al., 2017a). SWR events were subsequently identified as time windows starting at $1 \mathrm{SD}$, peaking at $>4$ $\mathrm{SD}$, and remaining at $>1$ SD for $>20$ and $<130 \mathrm{~ms}$ around the peak.

\section{LFP preprocessing}

Power bands were computed using multitaper spectograms (MTSpectrogram. $m$ in FMAtoolbox) between 0 and $250 \mathrm{~Hz}$ with a window of $2 \mathrm{~s}$, a step size of $50 \mathrm{~ms}$, and a time-frequency bandwidth of three and five tapers (Purpura and Bokil, 2008; Babadi and Brown, 2014). The spectral density powers are estimated in the following frequency domains: $\delta(0.5-4 \mathrm{~Hz}), \theta(7-14 \mathrm{~Hz}$ in hippocampus, $4-12 \mathrm{~Hz}$ in BLA), $\beta$ (15-30 Hz in hippocampus, $12-$ $30 \mathrm{~Hz}$ in BLA), low $\gamma(30-70 \mathrm{~Hz})$, high- $\gamma(70-120 \mathrm{~Hz})$, and, eventually, "ripples" and "fast" (both 120-250 Hz) for hippocampus and BLA, respectively (Buzsáki and Draguhn, 2004; Girardeau and Zugaro, 2011; Colgin, 2016; Bocchio et al., 2017). Band powers were computed for all the electrodes recording in the selected anatomical areas, i.e., right BLA and hippocampus. The $85 \%$ quantile value of their distribution was computed and used throughout the analysis. Note that the mapping of band powers to absolute values of time is irrelevant as long as all power time series are treated the same and as long as no other timeresolved quantities are present. The latter becomes relevant below, see Alignment settings.

\section{Topological causality (TC)}

TC relies on Takens' theorem and the theories of timelagged embedding that it forms the foundation of (Takens, 1981; Kantz and Schreiber, 2004; Bradley and Kantz,
2015; Harnack et al., 2017). A time-lagged embedding of dimensionality $m$ refers to the procedure where a time series is converted to a sequence of vectors in an $m$-dimensional space, and each dimension is defined by a specific time lag (see below for the mathematical formulation). In simple terms, Takens' theorem states that, given a partial observation of a dynamical system, e.g., of a time series of only one out of three effective variables in the system, a suitable embedding procedure will allow the (re)construction of an attractor preserving any true underlying attractor in a topological sense. This will hold if the dynamics are deterministic and couple the evolution of all underlying variables. TC exploits the theorem and its underlying theory, which is where the attribute "topological" derives from. Specifically, TC allows the quantification of the directed influence of two time series, which are assumed to be coupled with no requirement for linearity, on each other by essentially imposing Takens' theorem and measuring the consistency of the data with it. The numerical method to estimate it closely follows the one suggested in the original study (Harnack et al., 2017). The actual implementation starts by transforming the data points in the time series under investigation into their empirical cumulative distribution values (this is referred to as a quantile transformation in Harnack et al., 2017). This is done to achieve invariance of the TCs with respect to the actual distributions of the underlying sequences. In our case, the time series, i.e., band powers, were transformed separately within each behavioral epoch.

The actual TC values are derived as follows. Given a scalar variable $x$, the $m$-dimensional reconstructionspace vectors are constructed as $X(t)=\{x(t), x(t-\tau)$, ..., $x(t-(m-1) \tau)\}$. We chose the time lag $\tau$ as the $1 / e$ characteristic decay value of the average mutual information (Fraser and Swinney, 1986; Bradley and Kantz, 2015). Next, Sugihara's simplex projection method is applied to identify a suitable embedding dimension in a range of values from 2 to 10 (simplex in R package rEDM; Sugihara and May, 1990; Sugihara et al., 2012) where 10 turned out to be a sufficient upper limit in our case. $\tau$ and $m$ are obtained independently for each of the two time series $x$ and $y$ under investigation. In the actual embedding for the evaluation of TCs, each time series maintains its own $\tau$ while the actual $m$ used is the larger among the two (Cao et al., 1998; Arnhold et al., 1999; Hegger et al., 2000).

Given the embedded vector $X(t)$, the $k=2 m$ nearest neighbors are found. Here, we applied also the Theiler correction that excludes the points belonging to a temporal neighborhood of radius $\tau(m-1)$ from this search (Arnhold et al., 1999). The time indices of these $k$ neighbors are extracted and used to identify the respective projection of $X$ in the other embedded space $Y$. Let us denote as $\mathbf{K}_{x}$ and $\mathbf{K}_{y}$ the $k \times m$ matrices representing, respectively, the neighborhood of $X$ and its projection in the embedded space of $Y$. We wish to compute the local Jacobian matrix $J_{y \rightarrow x}^{t}$ of the mapping from $y$ to $x$. This is done by performing a principal component analysis $(\mathrm{PCA})$ on the joint matrix $\left[\mathbf{K}_{X}, \mathbf{K}_{Y}\right]$, followed by calculating $J_{y \rightarrow x}^{t}=\mathbf{P}_{X} \mathbf{P}_{Y}^{-1}$ where $\mathbf{P}_{X}\left(\mathbf{P}_{Y}\right)$ are the projections of $X(Y)$ onto the first $m$ principal components. The singular values $\sigma_{k, y \rightarrow x}^{t}$ of the Jacobian matrix yield the TC components as follows: 


$$
T C_{x \rightarrow y}^{t}=\frac{1}{1+\log \left(\prod_{k} \max \left(1, \sigma_{k, y \rightarrow x}^{t}\right)\right)} .
$$

In order to assess the statistical significance of the obtained value, we eliminate the notion of geometric neighborhood from the calculation. To do so, we select the $k$ projections of $X$ on $Y$ randomly and recompute the TC value. By repeating this $N=100$ times, we are able to generate a chance level distribution in the TC domain $[0,1]$. Because of the modest number of trials, which we were restricted to for computational reasons, a bounded density estimation with a $\beta$ kernel and a bandwidth equal to $\sigma N^{2 / 5}$ (R package bde; Chen, 1999) was adopted for increasing robustness. The true TC value was deemed significant if it was larger than the 95\% quantile of the chance level distribution, otherwise it was set to zero. The false discovery rate is controlled with the Benjamini-Yekutieli correction (Benjamini and Yekutieli, 2001), which was applied at every time step based on pooled data across the 72 TC indices (Sheikhattar et al., 2018).

The reconstruction of the manifold and the related computation of TC values presented below were performed within non-overlapping windows of 2-min length. The amount of independent data points depends on the rate of memory decay of the signals, and in our case the chosen interval of two minutes offered $\sim 100$ observations (see below). The details of the localization are also relevant for controlling the impact of non-stationarity issues along the trajectory, which can arise because of transient events, such as extraneous stimuli or experimenter intervention (Pesaran et al., 2018). This is particularly relevant in the run epoch where heterogeneous behaviors are present, but we refrained from attempting to investigate this quantitatively.

\section{Alignment settings}

Calculating the TCs and examining their values within specific time periods necessitated, to some degree, arbitrary choices regarding the temporal alignment between the different time series, namely, the band powers, TCs, and labels, e.g., NREM or SWRs. If we take the labels as reference for absolute time, this leaves two alignment choices to be made. Band powers were computed as multitaper spectrograms with windows of 2-s length, which were always center-aligned to the labels. The TC values, on their own, were computed in a lagged coordinate space, which corresponds to choosing a predictive embedding, i.e., for a given time point, the lagged data were taken exclusively from earlier times (for a pictorial description, see Extended Data Fig. 1-1). Clearly, the construction of spectrograms performs an averaging operation that will lead to some blurring in the localization of interactions. This effect should be kept in mind throughout. To investigate whether the analysis is robust with respect to the choice of alignment for these computed TC values, different alignments were analyzed systematically. TC embeddings were shifted, forward or backward, with respect to the time bins, and, thus, to the labels and power band windows. For each time bin, the $75 \%$ quantile over the 36 TCs was computed and, eventually, these values were averaged over the single sessions. As a global measure, the average of the unsigned differences between different time periods was computed (Extended Data Figs. 3-3, 3-4). Chance level distributions for each session were obtained by 1000 random block permutations of the TC values along the time axis (see below, Granger causality and cross-correlation functions), and the mean of the $95 \%$ quantiles across sessions was calculated.

\section{Granger causality (GC) and cross-correlation functions (CCFs)}

GC values were evaluated for the same set of time series used for the TCs by means of the MATLAB toolbox MVGC (Barnett and Seth, 2014). A variable $x$ is called "Granger cause" for $y$ if the predictability of a (chosen) model of $y(t)$ is improved by the inclusion of the history of $x$ with respect to the knowledge of the history of $y$ alone. GC values are computed from the ratio of the prediction error of the full (history of $x$ and $y$ ) and restricted (history of $y$ alone) models, respectively. The original and most common formulation adopts an autoregressive model; this is followed here as well (but see Discussion, Methodological and theoretical insights). In order to deal with non-stationarities and to allow a comparison with TCs, whose embedding length typically was in the range of a few seconds, GCs were calculated in sliding windows of 10-s length and with a step size of 1 s. A specific sleep phase, NREM or REM, was assigned to each value if its relative number of time frames in the integration window was larger than 50\% of the window size. The Bayesian information criterion was chosen to set the model order. Following regression and the calculation of the GC values, $p$ values were obtained from the theoretical asymptotic $F$ distribution (Barnett and Seth, 2014).

Pearson CCFs were computed in sliding windows of 2.5-s width and a minimum step size $(50 \mathrm{~ms})$. In order to test significance, for each BLA band power the related time series was divided into blocks of a size equal to its autocorrelation length. This was defined as the lag time needed to dip below an autocorrelation value of $1 / e$. The blocks were permuted, the CCFs were recalculated, and the whole procedure was repeated 200 times; $p$ values were extracted by comparing the null distributions thus obtained with the true values. Block permutation was applied to preserve the autocorrelation structure of the signal while disrupting the coordination with the other signals involved (Efron and Tibshirani, 1994; Chernick and LaBudde, 2014). The same technique was used in the analysis for other statistical tests involving time series, see above, Alignment settings and Extended Data Figures 6-1, 6-2.

As for TCs, for both GC and CCF values, the significance threshold was set to 0.05. The Benjamini-Yekutieli correction was applied, and values that were not statistically significant were zeroed explicitly. 


\section{Combined dataset of LFPs and TCs}

To combine the data, we convoluted the 72 TCs ( 36 in each direction) with an Epanechnikov kernel with 100-ms bandwidth. The 12 band powers from hippocampus and BLA were z-scored within each session. This allowed us to meaningfully bind the TCs and LFP band powers together in an $N \times 84$ matrix where $N$ is the number of time frames in all of the five behavioral epochs (pre-run, presleep, run, post-sleep, and post-run) of the available sessions (see above, Experimental design and behavior). Eventually, to eliminate redundancies across bands and across TC values, we performed a PCA. We selected a number of principal components (18) in proximity to the elbow point of the explained variance (Extended Data Fig. 4-8). When comparing to a dataset without the TCs, we used only the 12 band powers and no dimensionality reduction.

An alternative to the preprocessing of the combined dataset of TCs and band powers is offered by the adoption of locally adaptive weights (LAWs; Blöchliger et al., 2015). These weights are meant to correct for the fact that different time series may be of heterogeneous importance when the system is in different states. Briefly, at each time step we count the number of transitions across the global mean (per series) in a 5-s window centered at that point. The local value is then scaled by a quantity inversely proportional to this count such that time series that are mostly noisy get lower weights and are outweighed by those showing an effective metastable dynamics. It is important to note that the weights only modulate the importance of differences in time series. Thus, if there are no differences, the weights are irrelevant as, for example, for comparisons of different points where the TCs were are all zeroed explicitly. We then performed a PCA on the data matrix after scaling it by the LAWs and kept the first five components with the largest variances. When using the dataset without TCs, we applied the same procedure but only on the 12 band powers. This included PCA.

To test how much information the TCs provide relative to band powers, we performed two types of TC permutation tests. These permutations were applied within each session, i.e., before concatenation of all the sessions. In the first test, we shuffled only the time blocks containing TCs. This preserves the relative values of TCs for different band combinations but reassigns time in a blockwise manner. In the second, the TCs were permuted between each other within the same time frame. This preserves time but redistributes the 72 values to random power band combinations.

\section{Progress index (PI) and SAPPHIRE plot}

The PI analysis was applied to the dataset obtained from the preprocessing procedure described above (see Combined datasets of LFPs and TCs). For a full description of the method, we refer the reader to the literature (Blöchliger et al., 2013, 2014) and for a schematic illustration to Extended Data Figure 4-1A. Briefly, the method rearranges the time frames (snapshots) into a new order, called $\mathrm{PI}$. In the $\mathrm{PI}$, neighboring points are structurally similar in the feature space. To accomplish this, an initial snapshot is chosen a priori $(\mathrm{PI}=1)$. The snapshot to assign as $\mathrm{PI}=n$ (for $n>1$ ) is the closest to any member of the set of the already indexed $n-1$ snapshots. The algorithm continues progressively until all snapshots have been indexed. The addition rule corresponds to a singlelinkage criterion, and the (dis)similarity we employ is the Euclidean distance in the chosen feature space. In practice, the algorithm can be cast as the task of constructing the minimum spanning tree (MST), and the PI can be viewed as a particular order of the edges of the MST. Given the size of the datasets under investigation $(\sim 3 \times$ $10^{6}$ snapshots), we here resort to the approximate version of the MST, called short spanning tree (SST), as introduced in Blöchliger et al. (2013), which offers nearlinear scalability for the construction of the PI with respect to dataset size. Its construction relies on a hierarchical clustering technique (Vitalis and Caflisch, 2012), architecturally similar to the Balanced Iterative Reducing and Clustering using Hierarchies (BIRCH) algorithm (Zhang et al., 1996), whose parameters were tuned automatically according to ad hoc criteria. The initial $\mathrm{PI}$ snapshot $(\mathrm{PI}=1)$ is chosen as the center point of the largest cluster found by this preparatory partitioning. The PI sequence is then plotted against different labels and annotations in the so-called States And Pathways Projected with HIgh REsolution (SAPPHIRE) plot. A simple and helpful feature that can be plotted is the original time index (the little dots in the "Time" annotation). The second annotation to be derived purely from the time series is the "Kinetic" annotation, which measures the kinetic separation between regions to the left and to the right. In practice, given a snapshot with $\mathrm{PI}=n$, we call $A$ the set of points that lies, along the PI, within a window of length $L$ centered on $n$. We then count how many transitions along the time sequence happen between the left and right halves of $A$. In other words, focusing only on the snapshots within the interval $A$, we count how many times the time sequence jumps from the set of snapshots in the right half of $A$ to that in the left half and vice versa. The kinetic annotation (panel in the bottom) is proportional to the logarithm of the inverse of this count, also called cut function, and, thus, it assumes lower values when the snapshot $n$ lies close to the center of a cluster, and, conversely, higher ones when $n$ is located in a boundary region between different states. The window length $L$ was set to $10 \%$ of dataset size throughout the analysis. Only for visualization purposes, the kinetic annotation in the SAPPHIRE plots has been processed with a monotonic function to stress lower values and re-scaled between 0 and 1 .

The PI algorithm is implemented in the software CAMPARI (http://campari.sourceforge.net/). A wrapper of the original Fortran code has been used in the analysis ( $R$ package CampaRi). This is available from a public GitLab repository (https://gitlab.com/CaflischLab/CampaRi).

\section{Clustering from the SAPPHIRE plot}

We implemented a processing pipeline to transform the SAPPHIRE plot into a time-based clustering algorithm, called SAPPHIRE-based clustering ( $\mathrm{SbC}$ ), which is designed 
to extract clusters by partitioning the $\mathrm{PI}$ sequence into consecutive chunks. A detailed description, along with applications, is provided in Cocina et al. (2020) (but see Extended Data Fig. 4-1 for a schematic). A conceptually similar approach has been used in Garolini et al. (2019) for an identification of network states.

In essence, the $\mathrm{SbC}$ algorithm relies on both the kinetic and temporal annotations (the two panels in the bottom of the SAPPHIRE plot), which are first analyzed independently. For the kinetic annotation, we apply a simple peak identification algorithm to place candidate partitions along the $\mathrm{Pl}$. For the temporal annotation, we construct a 2D histogram (time vs $\mathrm{Pl}$ ) and parse it to identify horizontal stretches of consecutive bins with significant occupancies. Each of these represents an individual visit of a putative state in the original time series. Next, we extract a second set of putative partitions into clusters from the distribution of the boundaries of these stretches along the PI. This second set is pruned by a statistical test, which employs the Hellinger distance between the temporal distributions of adjacent stretches. The two sets of partitions, i.e., those derived from the kinetic and temporal annotations, respectively, are then merged (to eliminate redundancies), and the final trajectory clustering is delivered from this combined set.

The bin sizes in the 2D histograms on the PI axis, $w_{P I}$, and on the time axis, $w_{t}$, are the relevant parameters of the algorithm presented above (Extended Data Fig. 4-1B, panel 2). $W_{P I}$ sets the minimum size, and the resolution, of the clusters that we want to identify. On the other hand, $w_{t}$ should be of the order of the average residence time in the putative set of states. Overall, it must be recalled that the number of points in the temporal annotation is simply equal to the number of snapshots (time frames). Therefore, any combination of $w_{P I}$ and $w_{t}$ should be chosen such that the average density per bin is not too low where the threshold is set to be proportional to $w_{P I} w_{t}$. During the analysis, as a compromise between the above criteria and the final number of clusters, we chose $w_{P I}=300 \mathrm{~s}$ and $w_{t}=150 \mathrm{~s}$.

The $\mathrm{SbC}$ result is also used, in turn, for improving the SAPPHIRE plot itself. This is an extension to the original method as presented in Blöchliger et al. $(2013,2014)$. We compute a distance matrix between the clusters by taking the Hellinger distance mentioned before as a (pseudo) metric. This matrix is processed by a seriation algorithm to obtain a reordering of the clusters according to their reciprocal similarities (seriate in $\mathrm{R}$ package seriation; Hahsler et al., 2008; Behrisch et al., 2016). Finally, the PI chunks, i.e., the PI-continuous sets of snapshots representing the clusters, are reordered according to this new sequence by maintaining their internal arrangements, and the kinetic annotation is recomputed. All the SAPPHIRE plots shown in this work were postprocessed in this way.

\section{Other clustering methods}

Other clustering methods, which are commonly used for large datasets, were applied here for comparison. Specifically, we chose k-means clustering with mini batches (MiniBatchKmeans in the $\mathrm{R}$ package ClusterR) and k-medoids with either Euclidean or Manhattan distances (Aggarwal et al., 2001; clara in R package cluster). The number of clusters was always set equal to the one found by $\mathrm{SbC}$ for the dataset under investigation.

\section{Affinity scores}

Each cluster is given a set of affinity scores related to labels that describe the animal's state or behavior. For discrete variables, such as behavioral epochs (e.g., pre-sleep or run), sleep phases (i.e., NREM, REM, or wake), and the presence of SWRs, the number of occurrences of the label divided by the cluster size yielded the affinity score. For speed and firing rates, we made use of the average value within the cluster (ignoring any missing values).

The PreSleep and PostSleep labels generally refer to the periods where the rat is not located on the track and thus include awake phases. The Sleep label refers to both pre-sleep and post-sleep epochs but is restricted to time points annotated explicitly as either REM or NREM sleep phases; the remaining labels are equivalent to those shown in the SAPPHIRE plot.

\section{Matching of states across rats}

The clusters identified by $\mathrm{SbC}$ and the affinity scores related to these form the basis of a procedure that helps comparing SAPPHIRE plots across animals. In particular, we seek the clusters of Rat1 and Rat2 that are likely equivalent to the four recognizable basins (or coarse states) of Rat3. The similarity criterion adopted takes advantage of the affinity scores. The actual composition of the four basins in terms of $\mathrm{SbC}$ clusters (Extended Data Fig. 4-4, 93 clusters) was determined manually but straightforwardly by using the peaks of the kinetic annotation as guidance.

The affinity scores are contained in a matrix where the rows represent the clusters and the columns the different external labels. To account for differences in scale between the different labels but also across rats, we initially rank-transform the affinity scores across the clusters (i.e., along the rows) for each rat. For simplicity, we describe the procedure using Rat1 as the example (and the four basins of Rat3 as reference). We use the affinity scores to calculate the Pearson cross-correlation matrices between the clusters of Rat1 and those of Rat3. Next, each Rat1 cluster is associated to one of the four basins of Rat3, while trying to preserve the relative size of the four coarse states. Specifically, the cross-correlation matrix is collapsed into a list of triplets composed of cross-correlation values, the corresponding Rat1 cluster, and the implied Rat3 basin, which is the one containing that particular cluster of Rat3. This list is then sorted in descending order of correlation values and, progressively, each Rat1 cluster is assigned to the corresponding basin. If the relative size of the growing basin exceeds that size as seen for Rat3, or if the given cluster for Rat1 has already been assigned, the triplet is ignored, and no association is done. By performing this procedure for the entire list, all clusters of Rat1 are eventually assigned to one of the four basins. 


\section{Unfolding projection}

To visualize the results contained in the affinity scores in two dimensions, we resort to a multidimensional scaling (MDS) technique applicable to a generic rectangular matrix $\delta_{i j}$ with dimension $n \times m$ (Cox and Cox, 2000; Borg et al., 2018). The entries of the matrix are interpreted as the ranks that $n$ judges give to $m$ objects. The lower the rank the closer an object is to that judge's taste. The unfolding method consists in projecting both the judges and the objects onto a low-dimensional space. The projection tries to ensure that objects will be found closer to those judges who have rewarded them with an optimal rank. The low-dimensional configuration is found by minimizing Kruskal's stress function

$$
\sigma=\sum_{i=1}^{n} \sum_{j=1}^{m}\left(f\left(\delta_{i j}\right)-d_{i j}\right)^{2},
$$

where $d_{i j}$ indicates the new set of distances in the projected space, and $f(x)$ is a monotonic function. In order to remove dependencies on the size of the distance matrix and on the absolute magnitudes of its elements, the actual stress values shown in the analysis are normalized as follows:

$$
\sigma=\sqrt{\frac{\sum_{i=1}^{n} \sum_{j=1}^{m}\left(f\left(\delta_{i j}\right)-d_{i j}\right)^{2}}{\sum_{i=1}^{n} \sum_{j=1}^{m} f\left(\delta_{i j}\right)^{2}} .}
$$

We used the $\mathrm{R}$ package smacof for the analysis (de Leeuw and Mair, 2009; Borg et al., 2018). This type of unfolding was applied to the rectangular matrices of affinity scores (see above, Affinity scores, and Fig. 5A, panel 3). The judges are the labels, representing, e.g., NREM, REM, PostSleep, PreSleep, SWRs, and the objects are the clusters. For this, all affinity scores were converted into row(judge)-wise ranks and, then, projected onto a 2D space (MDS coordinates). We used a ratio transformation of distances $(f(x)=a x)$ and minimized the stress function (eq. 2) along with a penalty term. In order to check the robustness of the stress values, jackknife testing was performed by removing, in turn, a label (row) or a cluster (column) from the matrix of affinity scores.

The collective projection of all three rats is obtained by concatenating the rank-transformed affinity matrices of all the animals and performing the unfolding with the same settings presented before. Mean TCs were computed per cluster. Subsequently, we grouped TCs according to the leading band and averaged them within each group. For instance, we selected all the TCs driven by the BLA $\theta$ band, i.e., six values, and averaged them. Each of the obtained values constitutes a third dimension (TC) along with the two unfolding dimensions (MDS-1, MDS-2). In order to check whether the TC values are systematically distributed along the clusters, a plane was fitted in the [MDS-1, MDS-2, TC] space. The magnitude of the slope with respect to the [MDS-1, MDS-2] plane was extracted along with the orientation of the plane. These values can be represented by an arrow in the [MDS-1, MDS-2] space obtaining the so-called biplot.
The angles indicate the directions toward which the various TCs increase the most. The lengths of the arrows are proportional to the slope and quantify how well the TC values are mapped along the indicated direction. We used a permutation test to assess the significance of the value of the slope (same as in Kyriazi et al., 2018): TC values across clusters were permuted 1000 times, and a null distribution of absolute slope values was calculated. Only TCs for which the slope exceeded the $95 \%$ quantile of the null distribution were retained for the biplot.

\section{Statistical analysis}

All statistical tests were two-tailed tests unless stated otherwise. All tests comparing two sets of data were paired and non-parametric (Wilcoxon signed-rank test). Group data are reported with mean \pm SEM, unless stated otherwise. In cases of multiple hypothesis testing, we applied the false discovery rate correction according to Benjamini-Yekutieli to the individual tests' significance thresholds.

\section{Code and software accessibility}

Software used to carry out the analyses is mentioned throughout the text and is available online. Customized code and scripts supporting the current study are available at https://gitlab.com/CaflischLab/unsupervised_hpc-bla.

\section{Results}

To describe the LFP activity in the hippocampus and in the BLA (and subsequently to infer their reciprocal interactions), we used as time series the band powers computed with spectrograms on 2-s sliding windows with a 50-ms time step. We distinguish six different bands of physiological relevance: $\delta(0.5-4 \mathrm{~Hz}), \theta(7-14 \mathrm{~Hz}$ in hippocampus, $4-12 \mathrm{~Hz}$ in BLA), $\beta$ (15-30 Hz in hippocampus, $12-30 \mathrm{~Hz}$ in BLA), low- $\gamma(30-70 \mathrm{~Hz})$, high- $\gamma(70-120 \mathrm{~Hz})$, and, eventually, ripples and fast, respectively, for hippocampus and BLA (both 120-250 Hz; see Fig. 1D; Buzsáki and Draguhn, 2004; Girardeau and Zugaro, 2011; Colgin, 2016; Bocchio et al., 2017).

We have chosen the band powers to bridge distant frequency bands since they average the fluctuations in the signal across a shared time window. In this way, we homogenize the intrinsically heterogeneous timescales of variations in the LFPs across the different bands. Possible drawbacks of our approach are addressed in the Discussion section.

\section{Interaction analysis between behavioral epochs and sleep phases}

We use TC as a measure of directed interactions between the hippocampus and the BLA (Harnack et al., 2017). The method is based on a time-lagged embedding procedure derived from an underlying theory in the field of dynamical systems and nonlinear time series analysis (Kantz and Schreiber, 2004). Note that the term "causality" has a different notion in that field, and it is not directly related to the causality assessment exerting neural manipulations (more in Discussion). We provide here a conceptual explanation of the methods, and we refer the 
reader to the Materials and Methods section and to the original article for more details (Harnack et al., 2017). The time-lagged embedding consists in generating from a time series $x(t)$ a space of higher dimensionality $m$, called $X$, where the additional dimension corresponds simply to values of $x$ delayed by multiples of a time lag $\tau$; in explicit terms, $X(t)=\{x(t), x(t-\tau), \ldots, x(t-(m-1) \tau)\}$. Takens' theorem (Takens, 1981) stipulates that a lagged embedding of sufficient dimensionality is able to reconstruct the manifold generated by the underlying dynamical system (Kantz and Schreiber, 2004; Bradley and Kantz, 2015). In TC and our application of it, this implies that from the embedding of the hippocampal and BLA time series we should be able to extract relevant information regarding the reciprocal coupling of the two quantities. In particular, it should be possible to quantify to which degree the information of a time series is determined by the history of the other. A practical explanation is illustrated in Figure 1C. In the first example (upper panels) a case of unidirectional coupling is shown: $x$ influences $y$, that is, the time evolution of $y$ depends on $x$ but not vice versa. In the embedded space $X$, neighboring points of $X(t)$ are identified (blue circle) and the counterparts in $Y$, i.e., those with the same time indexes, are mapped graphically (straight lines from left to right). Since $x$ is fed no information from $y$ in this example, the points projected in $Y$ will be poorly "clustered" (i.e., the projected neighborhood diverges). When considering the other direction and, thus, mapping the neighborhood of $Y(t)$ (red circle) onto $X$, the projected points in $X$ will lie closer to each other since the evolution of $y(t)$ depended on $x$. In the second case (lower panel), $x$ and $y$ are reciprocally coupled, and both projected neighborhoods span compact areas. Generally speaking, the effective sizes of these neighborhoods will depend on the effective degree of influence of one time series onto the other. TC quantifies these as expansions, making it possible to derive a measure for the asymmetries in reciprocal influence between the two time series.

To be able to isolate significant directed interactions, the TC value at each time step is compared with the chance level distribution, which is obtained by mapping the variable's neighborhood randomly. TC values that do not pass this significance threshold are zeroed explicitly ( $p>0.05$, Benjamini-Yekutieli corrected; see Materials and Methods, Topological causality). Values of TC are computed between the band powers in the hippocampus and BLA (36 pairs, two directions). Importantly, despite the reliance on time windows for both spectrograms and manifold reconstruction ( $2 \mathrm{~s}$ and $2 \mathrm{~min}$, respectively), the resultant signals are able to resolve fast processes on the subsecond time scale, see Figure $1 E$. In the following, when discussing TC results, we will mostly use the terms "influence" or, more generically, "interactions" without referencing causality. We confront this terminological issue in detail in the Discussion section.

\section{Pronounced interactions between hippocampus and} amygdala occur with characteristic patterns during both NREM and REM sleep yet differ less between post-sleep and pre-sleep than between post-run and pre-run phases.

The mean values of TC during the sleep epochs are shown in Figure $2 A$. The distinction between NREM and
REM periods has been derived from the ratio between hippocampal $\delta$ and $\theta$ band powers (see Materials and Methods, Experimental design and behavior). During NREM, reciprocal influences between the low-frequency bands are prominent, in particular for the hippocampal $\delta, \theta$, and $\beta$ bands, as well as for the $\delta$ and $\theta$ bands in the BLA. To a lesser degree, $\delta$ and $\theta$ bands in the BLA appear to be driven by the hippocampal ripples band more than by the $\gamma$ bands. In addition, we observe that the BLA fast band is driven by hippocampal activity across the entire frequency range. During REM, some of the aforementioned interaction patterns decrease considerably, in particular those concerning the hippocampal $\beta$ band and those involving the low frequencies of the two regions $(\delta$ and $\theta$ ). A notable exception to this latter statement concerns the hippocampal, $\theta$-driven TCs, which are prominent and match the expected characteristics of REM sleep. These TCs are strong relative to most of the BLA frequencies and are complemented by BLA-driven TCs on the two highest frequency bands. The TCs found for the hippocampal $\theta$ band are mirrored partially in the high- $\gamma$ band. Based on the BLA-driven TCs in the high-frequency bands, there does appear to be some reciprocal communication during REM. The differences between post-sleep and pre-sleep epochs (Fig. 2A, right panel) are generally much lower than those between sleep phases (Fig. 2A, bottom panel; Wilcoxon signed-rank tests, $n=16)$. One exception to this regards the $\beta$ band of the BLA where two TC differences are significant. This contrasts sharply with the analysis performed on pre-run and post-run epochs. Here, a marked enhancement of TC values is visible during the post-run epoch (Extended Data Fig. 2-1; Wilcoxon signed-rank tests, $n=17)$. This difference is prominent for most frequency bands and in both directions. Changes in the influence exerted by the hippocampal $\theta, \beta$, high- $\gamma$, and ripples bands are pronounced in the safe running direction but somewhat weakened, for the $\theta$ and ripples bands, in the aversive direction. Conversely, interactions exerted by the BLA on the hippocampus are strengthened in post-run epochs in the aversive direction: $\delta$-driven and, especially, $\theta$-driven interactions are particularly enhanced. We stress that the aversive stimulus is absent in the postrun epoch, so the differences in Extended Data Figure 2-1 are likely related to the memory retrieval of the previous conditioning experience.

Apparent interactions between brain regions can also result from volume conduction, both incoming from external regions or, specifically, from hippocampus to BLA. This mechanism might contribute to any signals suggesting interactions involving low-frequency bands like $\delta$ and $\theta$ (Łesski et al., 2013; Bertone-Cueto et al., 2020). Regarding the $\theta$ band, however, several pieces of evidence of both behavioral and biological nature suggest that volume conduction is unlikely to be a major factor; these are summarized in Pape and Pare (2010) and Bocchio et al. (2017). For example, it has been observed that activities in the $\theta$ band of the lateral amygdalar and 

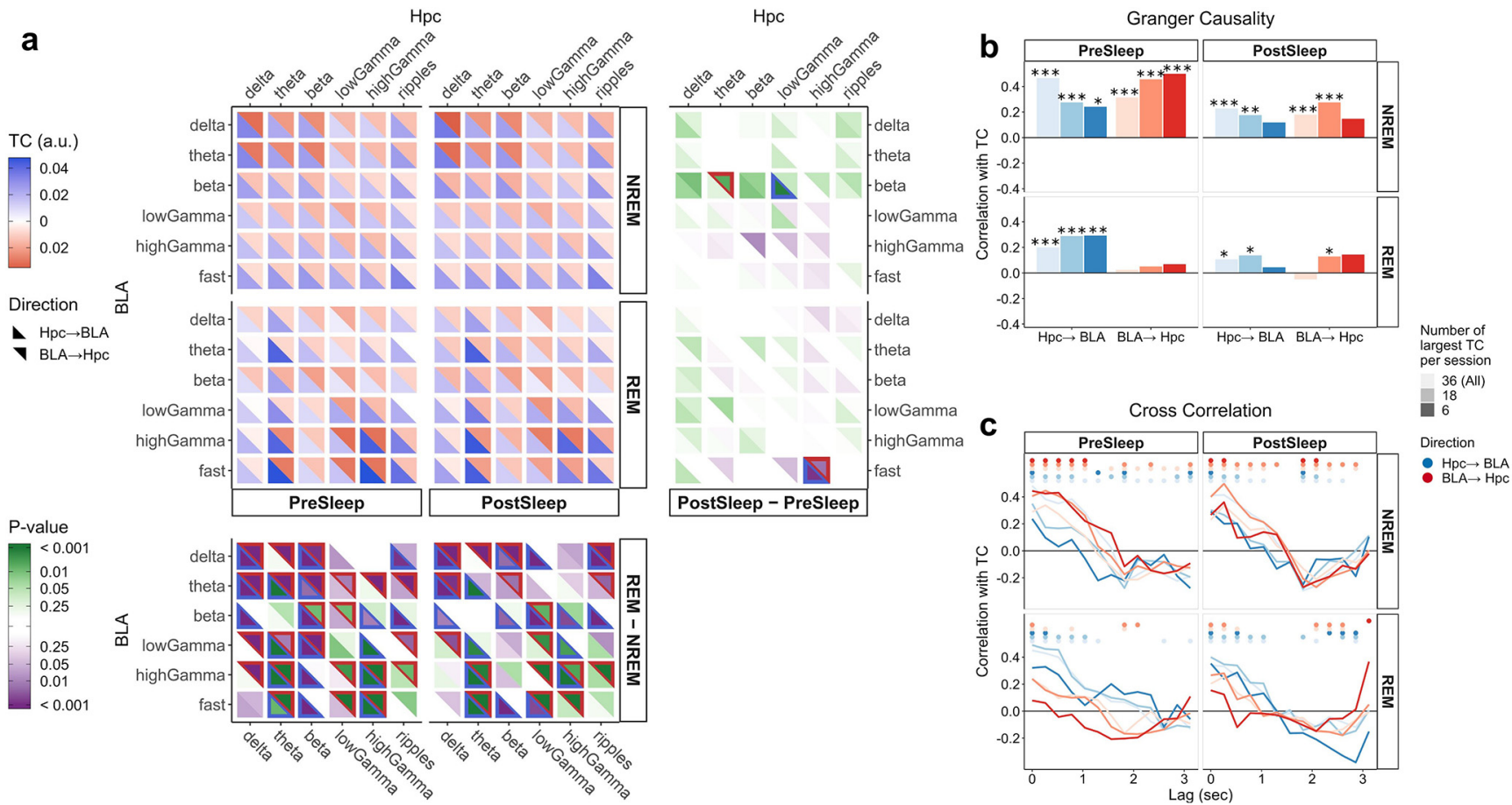

Figure 2. Reciprocal influence of hippocampus and BLA during sleep. $\boldsymbol{A}$, TC results in interaction tables. Central panels, Mean values across all the sessions ( $n=16,3$ rats) of the average TC values per sleep epoch and the two annotated sleep phases (NREM and REM). All sessions included in the calculation feature at least 1 min of total REM sleep in both pre-sleep and post-sleep phases. Every square shows two values for a given combination of frequency bands: the directions of influence are indicated by both color and placement of the two triangles (blue and lower-left for hippocampus $\rightarrow$ BLA, red and upper-right for the reverse). To aid visibility, the minimum value across the four panels and both directions was subtracted for plotting. Bottom and right panels, $p$ values of the two-tailed Wilcoxon signed-rank test on the differences of the mean values shown in the central panels. The right panels show test results for differences between sleep epochs, and the bottom panels those for differences between sleep phases. Color hue is given by the sign of the test statistic (value of zero under the null hypothesis). Given a comparison of the type $A-B$, green colors indicate higher values in $A$ relative to $B$. The triangle shapes are outlined when the corresponding $p<0.05$. NREM was distinguished from REM sleep by the hippocampal $\delta / \theta$ ratio (see Materials and Methods, Experimental design and behavior). The same analysis was performed for pre-run and post-run epochs when distinguishing between safe and aversive direction (Extended Data Fig. 2-1). To examine a possible impact of volume conduction on TC values, phase differences between the LFPs of the two areas are shown in Extended Data Figure 2-2. B, Comparison of TC with GC. For each combination of sleep phases, epochs, and direction of influence, mean values of GC were computed. For each of the $n=16$ sessions, in each direction, the largest $k$ (out of 36) TC values were retained and compared with the corresponding GC ones. Their similarity was quantified by computing Pearson correlation coefficients between the two $k \times n$ matrices. Asterisks indicate significant correlation values (Student's $t$ test, ${ }^{*} p<0.05$, ${ }^{* *} p<0.01$, $\left.{ }^{\star \star \star} p<0.001\right)$. C, Comparison of TC with CCFs. The same procedure described above for GC was performed for different values of the CCF time lag. In CCF, the frequency band leading in time is considered as the driver of the interaction. Points on the upper part of the plots denote significance of the respective correlation value (Student's $t$ test, $p<0.05$ ).

CA1 hippocampal regions are synchronized during fear memory retrieval but not during exploratory behavior (Narayanan et al., 2007; Seidenbecher et al., 2003). To estimate the potential influence of volume conduction on our analysis, Extended Data Figure 2-2 shows computed phase differences for LFPs filtered to the $\delta$ and $\theta$ bands between the two anatomical areas in different time epochs. While phase differences close to zero would correspond to expectation for volume-conducted oscillations (Nolte et al., 2004), the histograms are generally broad and not consistently peaked at zero phase difference for the $\theta$ band. This corroborates the aforementioned hypothesis that volume conduction plays a negligible role in this band. For the $\delta$ band, phase differences do consistently show the highest values in the proximity of zero in Extended Data Figure 2-2. While this does not need to result from volume conduction, the data in conjunction with literature observations of volume conduction in this frequency band in nearby regions (Bertone-Cueto et al., 2020) suggest a more cautious interpretation for $\delta-\delta$ interactions.

\section{Two independent measures correlate with TC if the mem- ory lengths are matched.}

To assess the robustness of our analysis, we next compared TC with two common linear techniques used to investigate directed interactions between oscillatory activities and connectivity between distant brain regions. Specifically, we chose GC and CCFs with varying time lag. We restricted ourselves to the same four data subsets shown in Figure 2A. GC and CCF values were computed for all the 36 frequency band combinations. This was done separately for both directions of influence. For each 
session and time period, we selected the largest 6, 18, or 36 (i.e., all) TC values and calculated their Pearson correlation with the respective $\mathrm{GC}$ and $\mathrm{CCF}$ values. $\mathrm{GC}$ and $\mathrm{TC}$ are compared in Figure $2 B$, which highlights that there are significant levels of (linear) correlation for the data from the NREM phase. These tend to be generally lower for post-sleep with respect to pre-sleep, in particular when selecting a lower number of frequency band combinations. In REM, GC and TC values are generally uncorrelated for the BLA $\rightarrow$ hippocampus direction, and, as for NREM, pre-sleep patterns display higher correlations than post-sleep ones. In Figure 2C, the comparison of TC values with CCF values with time lags of up to $\sim 3 \mathrm{~s}$ is presented. The emerging picture is very similar to that seen for the TC/GC comparison if short time lags of $<1 \mathrm{~s}$ are considered. For data taken from REM sleep, correlations of BLA-driven interactions are slightly more erratic as seen from the dependence on the number of variable pairs considered and the lower significance of the results (circles on top). Moreover, TC and CCF values start to anti-correlate almost everywhere when the time lag exceeds a threshold of $\sim 1-1.5 \mathrm{~s}$. This value is comparable to the average embedding length determined for TCs, which depends on both a base lag and a choice of dimensionality (see Materials and Methods, Topological causality). Why do TC values correlate only weakly or erratically with GC/CCF values if the source data are restricted to the REM period? First, it is important to point out that REM sleep is characterized by shorter episodes than NREM. In this case, the assignment of the REM label to the computed value, either TC, GC, or CCF, can be ambiguous given that each technique has its own different integration window that may extend across adjacent NREM time frames. Second, the TCs in the BLA $\rightarrow$ hippocampus direction during REM are confined to the fast frequency bands, and the underlying activities might be too short-lived to be picked up by a correlation measure. Third, both classical GC (as used here) and CCF are intrinsically linear techniques whose application to nonlinear systems may lead to equivocal or misleading results (Sugihara et al., 2012; Harnack et al., 2017; see Discussion, Methodological and theoretical insights).

SWRs are associated with unidirectional communication from the hippocampus to the amygdala during NREM sleep and awake phases.

Because of the results shown in Figure $2 B, C$, we focus for the following analysis only on NREM sleep and on the potential role of SWRs for the modulation of interaction patterns. To answer this question, we partitioned the NREM data into subsets featuring SWRs and those that do not (inter-SWRs; see Materials and Methods, Experimental design and behavior for the procedure used to detect SWRs). From Figure 3, we can glean that there is a broad influence of the hippocampus on the BLA driven by its ripples band and, to a lesser extent, by its low-frequency bands, including $\delta, \theta$, and $\beta$. While these hippocampal drives are enhanced during SWR phases, the reciprocal BLA-driven interactions seem to not be affected strongly by the SWRs (Wilcoxon signed-rank tests, $n=20$ ). This suggests the interpretation that SWRs either facilitate or are at least a hallmark of communication going out from the hippocampus. Interestingly, although SWR phases show higher TC values overall for hippocampus $\rightarrow$ BLA, significant differences between pre-sleep and post-sleep phases are more commonly found for data from time periods devoid of SWRs.

We also investigated the variation in TCs with respect to the presence of SWRs both during the wake periods in pre-sleep and post-sleep epochs (Extended Data Fig. 31), and during the run epoch itself (Extended Data Fig. 32). In the former, with respect to NREM, SWRs are associated with significantly increased interactions between BLA $\delta$ and $\theta$ bands and most bands of the hippocampus (Wilcoxon signed-rank tests, $n=20$ ). On the other hand, signal inputs from the BLA to the high-frequency bands of the hippocampus are weakened considerably. Instead, during the actual run epoch (Extended Data Fig. 3-2), the modulation by SWRs is much less pronounced overall: it appears to be restricted primarily to hippocampus-driven interactions in the ripples band (Wilcoxon signed-rank tests, $n=13$ ). Furthermore, TC values during either SWR intervals or in between do not seem to distinguish the running directions strongly. The clearest recognition appears to come from BLA-driven processes, slightly more so in the absence of SWRs. These enhancements found when the rat encounters the aversive stimulus could be related to the stimulus itself or to fear conditioning. We also investigated how the relative alignment between TC and SWR labels can affect the analysis. Results shown in Extended Data Figures 3-3, 3-4 support our adopted settings (see also Materials and Methods, Alignment settings).

\section{Global interaction analysis of data-derived, neural states}

Similar patterns of directed influence, reciprocal or not, appear across different epochs, different sleep phases, or different behaviors. These patterns can reveal information that goes beyond the annotations usually employed to characterize the animal's state, e.g., running speed or neuronal firing rates. We provide a methodology to assess whether TCs, along with band powers, can help to reveal putative states related to activity in the hippocampus, the $B L A$, and the coordination between them. For this purpose, we use, for each session, the entire time series without selecting any particular epoch of interest a priori, i.e., without discarding periods that may be deemed irrelevant. In detail, we merged the time series of TCs and the LFP band powers. The resulting joint dataset contains 84 time series: 6 power bands each for hippocampus and BLA and 36 TC values in either direction of interaction. These data had to be preprocessed to feasibly combine the two different classes of time series. The projection of the resulting dataset onto its first 18 principal components was used for further analyses (see Materials and Methods, Combined dataset of LFPs and TCs).

Our first goal is to highlight and annotate putative neural states that are visited recurrently (in time) throughout the experiment. To accomplish this, we use an unsupervised 

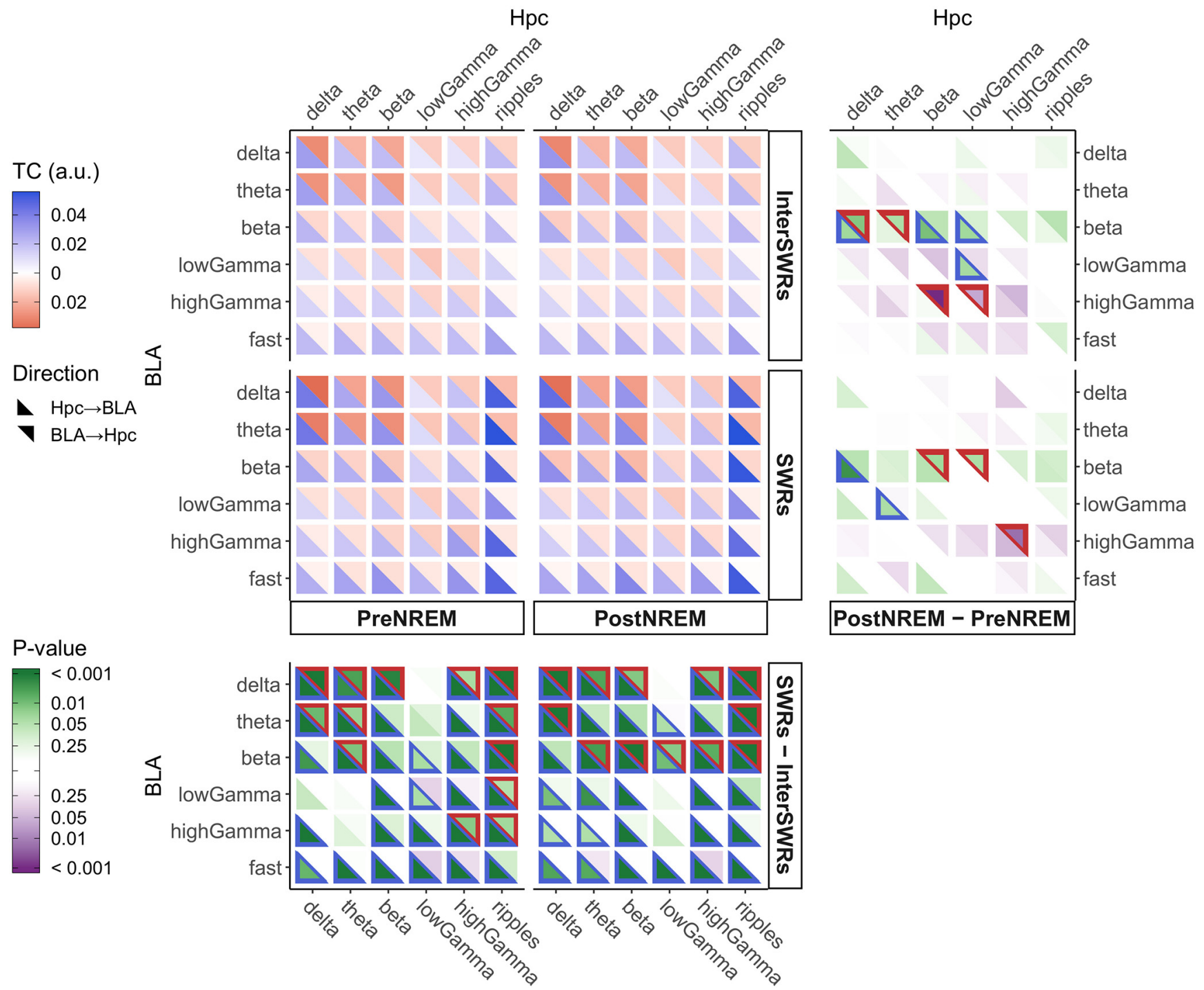

Figure 3. TC analysis during NREM sleep. Following the same procedure as for Figure $2 A$, we display TC results for pre-NREM and post-NREM sleep in the presence or absence of SWRs $(n=20,3$ rats). All sessions included show at least $5 \mathrm{~s}$ of total time featuring SWRs in both the pre-NREM and post-NREM epochs. The analogous figures for the wake phases and for the run epochs are shown in Extended Data Figures 3-1, 3-2, respectively. TC differences are robust to the choice of alignment between TCs and SWRs timestamps (Extended Data Flgures 3-3, 3-4). See details in Materials and Methods, Alignment settings.

algorithm that reorders the time series as the so-called PI (Blöchliger et al., 2013). From this reordering, we obtain with the help of suitable annotations a SAPPHIRE plot (Blöchliger et al., 2014). These scalable techniques were originally developed for the analysis of molecular dynamics simulations, but their applicability is general for time series data (Blöchliger et al., 2013), as shown also in (Klein et al., 2017). While the details can be found in the Materials and Methods section, we provide a short rationale next (see also Extended Data Fig. 4-1). A state is a collection of time points that have similar properties. Unless specified otherwise, these properties are the aforementioned 18 principal components (of the 12 band powers and 72 TC values). With respect to our data, the terms "clusters" and "states" are used interchangeably given that the latter are identified by a clustering method. The reordering into the $\mathrm{Pl}$ attempts to group all instances of mutually similar time points, which may occur at vastly different times, into the same neighborhood along the PI. The peaks in the kinetic profile help to identify areas with few transitions between adjacent PI segments, i.e., they suggest the boundaries by which to delineate different states (Fig. 4, bottom panel). The original temporal index, along with time series and labels which may or may not be part of the original dataset, are then reordered according to the PI sequence and plotted on top of the kinetic profile. In this way, they provide further recognition of states and offer an initial characterization.

\section{Neural states at coarse resolution isolate both sleep} phases and emotional stimuli.

Results for Rat3 are shown in Figure 4, while those for the other two animals are displayed in Extended Data 


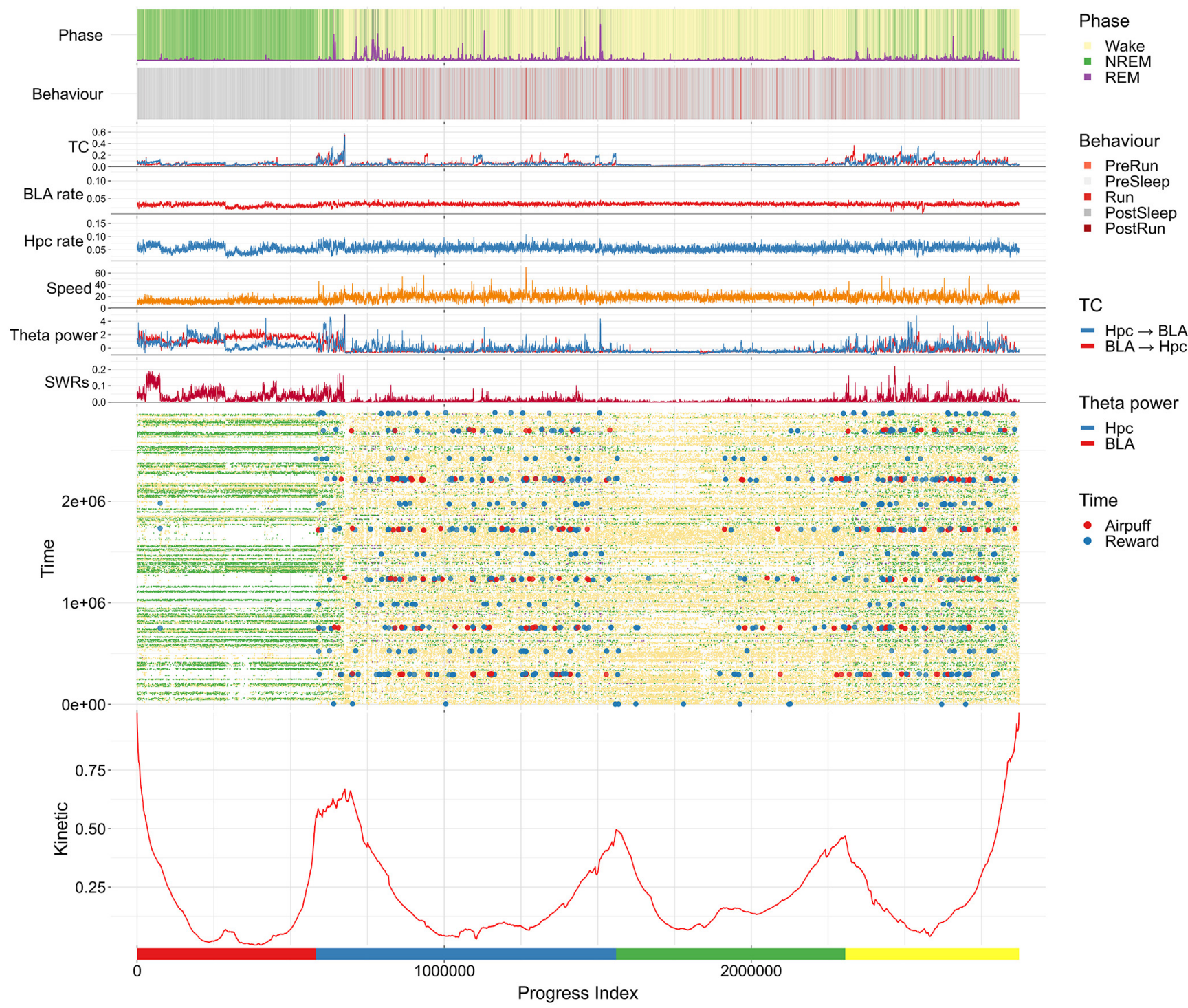

Figure 4. SAPPHIRE plot of the combined dataset of band powers and TCs. We show the results for Rat3 (6 sessions; all five behavioral epochs included; $\sim 40 \mathrm{~h}$ in total; 50 -ms resolution). The time series was rearranged according to a similarity criterion in the socalled PI (see Materials and Methods, Progress index (PI) and SAPPHIRE plot, and also Extended Data Fig. 4-1A for a schematic illustration). The kinetic annotation (bottom, red curve) highlights transitions between putative states. Directly above, the original position along the time sequence is plotted as a function of the PI. The color code is given by the sleep phase (small dots, see "Phase" legend) and by the type of events encountered while awake (circles, see "Time" legend). Because of limits to plotting resolution, a regular subsampling by a factor of $\sim 10$ was applied along the PI dimension. In the five panels above the Time annotation, we show, respectively, the histogram of SWRs, the z-scored $\theta$ powers of both regions (thresholded at 5), movement speed, firing rates of pyramidal neurons in hippocampus and BLA, and the average TCs in both directions. These six features were smoothed with a moving average filter employing a window of 500 points along the PI before plotting. The scales on the $y$-axes of these six annotations are homogenized across all SAPPHIRE plots. Finally, the two topmost panels indicate by color sleep phases and behavioral epochs, respectively (legends on the right). To be able to spot the REM phase more easily, the top panel is augmented with a curve indicating the fraction of REM-labeled points on a sliding window of $25 \mathrm{~s}$. Note that the pre-run and post-run epochs form just $\sim 2 \%$ of the overall time series, thus making them difficult to distinguish in the plot. At the very bottom of the plot, colored bars indicate four coarse states. Maintaining this color scheme, a matching procedure between these coarse states and putative ones in the remaining two rats (Extended Data Figs. 4-2, 4-3) was applied (Materials and Methods, Matching of states across rats) to facilitate comparisons across animals. Extended Data Figure 4-4 shows the same SAPPHIRE plot highlighting the clusters derived from SbC (see text and Extended Data Fig. 4-1B). We applied two different preprocessing pipelines to the combined dataset of TCs and band powers, which differ most strongly in the number of principal components retained, specifically, 5 and 18 (see details in Materials and Methods, Combined dataset of LFPs and TCs). The SAPPHIRE plots presented here and in Extended Data Figures 4-2, 4-3 for the remaining rats are for the dataset with 18 dimensions, see also Extended Data Figure 4-8; the results obtained with the alternative preprocessing (5 components) are shown instead in Extended Data Figures 4-5, 4-6, 4-7. 
Figures 4-2, 4-3. Four coarse states (basins) can be recognized with ease from the combination of the different annotations (Fig. 4, colored bars at the bottom). From the kinetic annotation (bottom) and the sleep phase annotation (top), we find that most of the NREM sleep data points are found in the first large coarse state (basin) on the left. The remainder of the NREM-labeled time points appear in the transition area immediately to the right of this basin as well as in the rightmost one. Time frames from NREM sleep and awake periods are interspersed in these two areas presumably because they share bidirectional and unidirectional TC patterns ("TC" annotation). On the other hand, REM-labeled points appear in the second and, to a lesser extent, in the last basin, along with time points when the animal was awake. Consistent with Figure $2 A$, these two regions show two different degrees of TC activity, that is, lower in the second coarse basin from the left and higher in the rightmost one. Air puff and reward times (the circles in the Time annotation) are mostly scattered throughout the two large basins that display noticeable TC values. Interestingly, the SWRs ("SWRs" annotation) mirror almost exactly the same pattern as the TCs except in the leftmost NREM basin indicating an apparent decoupling of the BLA from the hippocampus during NREM sleep. A similar observation can be made for power in the $\theta$ band ("Theta power" annotation): high but differing band powers between hippocampus and BLA are associated with lower TC levels (leftmost basin), whereas lower, but seemingly more synchronous band powers are associated with higher interaction values (rightmost basin). Stimuli encounters are noticeably absent from the third basin, which is homogeneous in neuronal activities and movement ("BLA rate," "Hpc Rate," and "Speed" annotations) but shows no sign of significant SWRs and TCs.

To aid comparisons across animals, we devised a matching procedure that pairs $\mathrm{PI}$ regions of Rat1 and Rat2 (Extended Data Figs. 4-2, 4-3, bottom bars) to the four basins of Rat3 (colors are matched). The procedure is based on similarities between the external annotations, e.g., "NREM" or "Hpc rate", and relies on the clusters identified from the SAPPHIRE plots (Materials and Methods, Matching of states across rats). These elements will be described in detail below. For Rat2 (Extended Data Fig. 4$3)$, the association provides a landscape similar to Rat3 for the red and green basins, with a mixing between the TC-active yellow and blue states in the intermediate PI regions. Conversely, the data from Rat1 (Extended Data Fig. 4-2) give rise to basins that correspond less to those of Rat2 or Rat3. Instead, despite the emergence of similar feature patterns visible in the TC and SWRs annotations, the algorithm appears to identify many smaller substates with sometimes very peculiar features (e.g., the tiny leftmost state). It is worth mentioning that Rat1 not only showed a reduced rate of crossings in the run epochs compared with Rat2/3 $(\sim 0.3, \sim 1.0$, and $\sim 2.0$, respectively) but also exhibited a $\sim 30$-fold difference in this rate across sessions ( $\sim 2-$ to 3 -fold for Rat2/3). This can be recognized clearly in the SAPPHIRE plot (Extended Data Fig.
4-2) from the lower density of air puffs and reward points in the Time annotation. The SAPPHIRE plot for Rat1 (Extended Data Fig. 4-2) reflects this variability in behavior. We stress that the matching algorithm is simplistic, in particular because of imposing a size constraint derived from Rat3 (see Materials and Methods, Matching of states across rats). We also emphasize that the order of states in a SAPPHIRE plot is arbitrary. The role of the four-color highlighting is primarily to locate corresponding regions across animals quickly. Generally speaking, and this holds for all three animals, the time annotation shows that the individual experimental sessions all contribute roughly equally to a given coarse state although the recordings are almost certainly non-stationary across days because of the progressive lowering of the electrodes (see Materials and Methods, Experimental design and behavior). Importantly, within all of these aforementioned coarse states, the kinetic annotation suggests potential substates, and this is what we investigate next.

\section{Clustering by the SAPPHIRE plot and characterization of the identified states.}

The SAPPHIRE plot provides a comprehensive visualization of putative states along with the labels used to characterize individual points in time. Nonetheless, given the size of the dataset and the amount of labels to be considered at the same time, we deemed it desirable to compress the amount of information displayed. Toward this goal, we take advantage of the kinetic and time annotations to formally identify clusters consisting of time points that are both kinetically and structurally close. The method is called $\mathrm{SbC}$ given that clusters originate directly from the partitioning of the PI sequence into chunks (see Cocina et al., 2020; Materials and Methods, Clustering from the SAPPHIRE plot, as well as Extended Data Figs. 4-1, 4-4). Specifically, we combine the PI ordering with the temporal information as found in both the Kinetic and Time annotations. In the former, the partitions are identified as peaks in the Kinetic curve, whereas in the latter "blocks" of distinct temporal patterns are identified from an underlying 2D histogram (Extended Data Fig. 4-1B, panels 2, 3). Eventually, a consensus is reached across the results from the two annotations. The number of clusters cannot be set directly but is controllable monotonically through the size of the bin in the aforementioned histogram, which sets an upper limit for the resolution of small clusters (see Materials and Methods, Clustering from the SAPPHIRE plot and Cocina et al. (2020) for more details). It is important to note that we exclude external annotations, e.g., the labels on the sleep phases, to arrive at the partitioning. Given that the original dataset of TCs and band powers is what defines the PI ordering, we can characterize each extracted cluster by a particular combination of internal state values, i.e., the LFP power bands and reciprocal interaction patterns between hippocampus and BLA (Fig. 5A). Of course, the definition of a single fingerprint per cluster masks the heterogeneity within it, which means that a relatively fine partitioning is required for these fingerprints to be informative. 
Once the clusters have been identified, we assign, to each of them, an affinity score related to the labels that annotate the SAPPHIRE plot (Fig. 4). These results are plotted with the help of the unfolding method, a dimensionality reduction technique that can project both clusters and labels onto a 2D space (Cox and Cox, 2000; Borg et al., 2018). This procedure, in good part inspired by previous traditional MDS applications (Shinomoto et al., 2009; Kyriazi et al., 2018), provides an effective visualization of the distribution of the states across the different labels. The unfolding method is an MDS technique in that it aims to minimize a stress function that accounts for the differences between the distance matrix in the original space and the one in the reduced space (see Materials and Methods, Unfolding projection). It is worth clarifying how the distance matrices differ between the unfolding technique and traditional MDS. In the latter, one would compute, e.g., Euclidean, distances between $n$ clusters using affinity scores for individual labels as coordinates in an $\mathrm{m}$ dimensional space, thus projecting only the clusters onto the reduced space. In the unfolding case, the $n$ clusters and $m$ labels are both considered as points to be projected. A distance matrix is assembled that relies only on affinity scores to control the placement of clusters relative to labels but does not supply data to the stress function that contains information about distances among clusters or among labels.

Unfolding projections for the three rats are shown in Figure $5 B-D$. Across all three animals, the first thing to note is that there are two (nearly) consistent clusters of labels: Speed, Run, and Wake versus NREM, Sleep, PostSleep, and SWRs. The remaining four labels (Hpc Rate, $\mathrm{BLA}$ rate, REM, and PreSleep) are more volatile. To understand the placement of clusters relative to labels, it is important to realize that the labels are not mutually exclusive, e.g., NREM must coincide with Sleep and spans both PreSleep and PostSleep labels. Thus, it is impossible for a cluster to coincide exactly with a single label. This limitation leads to the observed distribution, which is fairly uniform across the plot. However, we can assert that for at least two rats the placement does show an affinity with specific labels, which is highlighted by the coloring according to PI position as seen, in particular for Rat2 and Rat3, by comparing the SAPPHIRE plots of Extended Data Figure 4-3 and Figure 4 with the unfolding projections of Figure $5 C, D$, respectively. For these two cases, the clusters from the left of the SAPPHIRE plots (Fig. 5C,D, light colors) are clearly associated with NREM phases as expected from Extended Data Figure 4-3 and Figure 4. In addition, for Rat2 there is an accumulation of clusters shown in gray near the REM label (Fig. $5 C$ ), which is consistent with the higher prevalence of REM time points in the intermediate regions of the PI of Extended Data Figure 4-3. In all three panels, the layout suggests that the first dimension (horizontal) resolves a transition between awake/active and deep-sleep (NREM) phases. Since such a distinction can be inferred by the power band values alone, we conjectured that the second dimension might be related to the overall level of TCs. In order to assess this in a collective picture for all the rats, we performed the unfolding on the dataset combining all three affinity matrices. A so-called biplot is shown in Figure $5 E$ where the direction of enhancement of TC is indicated by arrows (see Materials and Methods, Unfolding projection). Each arrow indicates the average influence of individual bands, pointing toward the direction of larger enhancement across the low-dimensional space. The TCs that are significantly mapped in reduced space point downwards and overlap, especially for most of the hippocampus $\rightarrow$ BLA interactions. This confirms that "MDS-2" (i.e., the vertical dimension) is predominantly an overall interaction axis (high at the bottom, low at the top). In conjunction with the placement of labels, this is consistent with the results presented above (see Interaction analysis between behavioral epochs and sleep phases).

\section{When quantifying the ability to separate labels, the SbC method shows the highest fidelity among four tested methods and TCs effectively contribute to a better classification.}

It is useful to question whether the analysis in Figures 4, 5 yields putative clusters that do in fact describe the internal state of the joint hippocampus-BLA system. Unfortunately, in the absence of a ground truth, the only feasible way to gather evidence for this is to quantify how well states tend to separate labels, e.g., behavioral annotations. Here, our proxy for performance are the stress values from the unfolding technique, which measure the degree of both the quality of the projection and the ability of the state partitioning to sort out the different labels (see Material and Methods, Affinity scores). Extended Data Figure 6-1 shows that the volatility of certain label positions in Figure $5 B-D$, in particular BLA rate and PreSleep, is mirrored in their high relative stress contributions for Rat1 and Rat2. Generally speaking, most labels appear to make similar contributions to the stress, however.

Such a global measure of performance also allows us to compare the clustering obtained by $\mathrm{SbC}$ to those obtained with other methods. In Figure $6 A$, results from this comparison are shown for three clustering techniques commonly used for large datasets. In all cases, the number of clusters was set equal to that obtained with the $\mathrm{SbC}$ method since stress values tend to depend on the number of points to be projected. As an additional test, we were also interested in the effective contribution of the TCs in separating the labels. To do so, we performed the $\mathrm{SbC}$ algorithm on a dataset not containing TCs. The data show that the $\mathrm{SbC}$ achieves lower (i.e., better) stress values with respect to the other clustering methods. We also note that neglecting TCs results in poorer performance in two rats and in comparable values for the remaining animal, indicating that TCs are helpful in deciphering the coding in these brain areas. It is a caveat that the number of clusters differed slightly (it is not a direct parameter in the $\mathrm{SbC}$ algorithm, see Materials and Methods, Clustering from the SAPPHIRE plot). The relevance of TCs collected as a function of time was examined in two random permutation tests (Fig. 6B). In the first, the time bins of TCs were shuffled in blocks, which maintains their local patterns but mismatches them with the band powers' time points. In the second, we permuted the TC identity at each time bin 
a

1

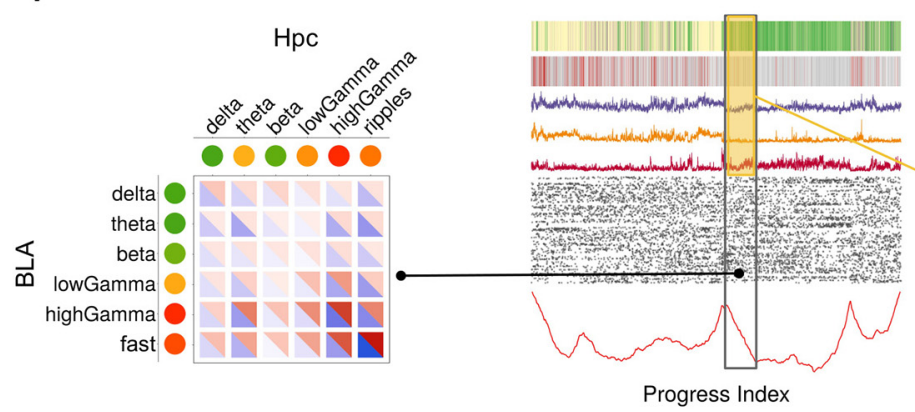

3

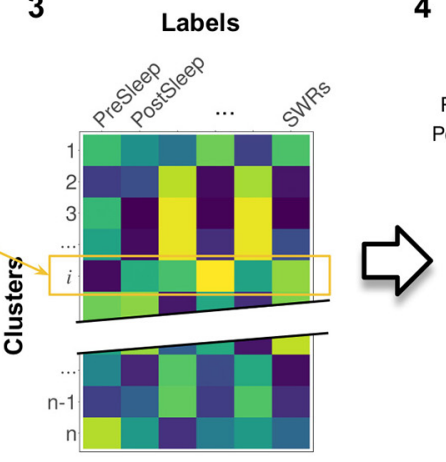

4

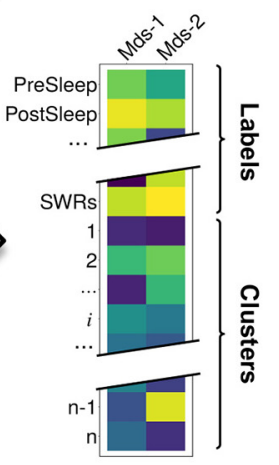

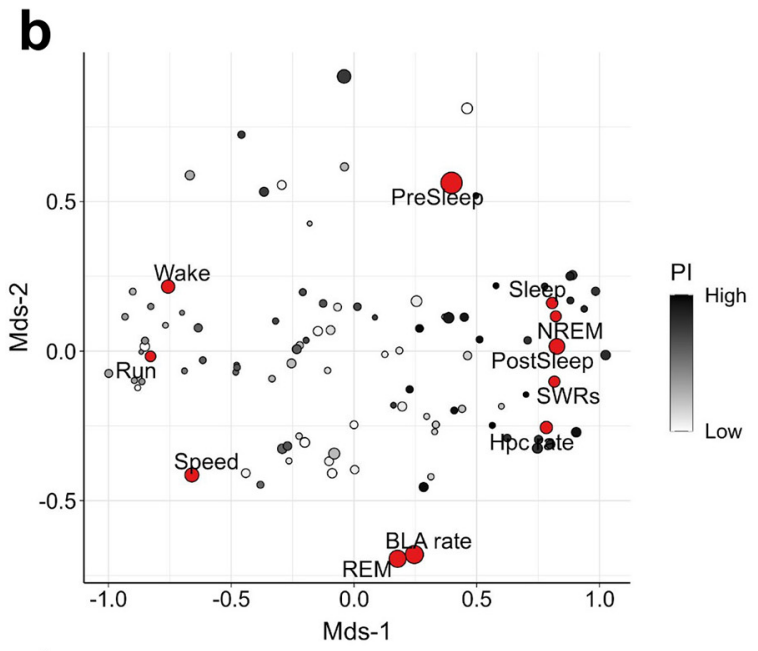
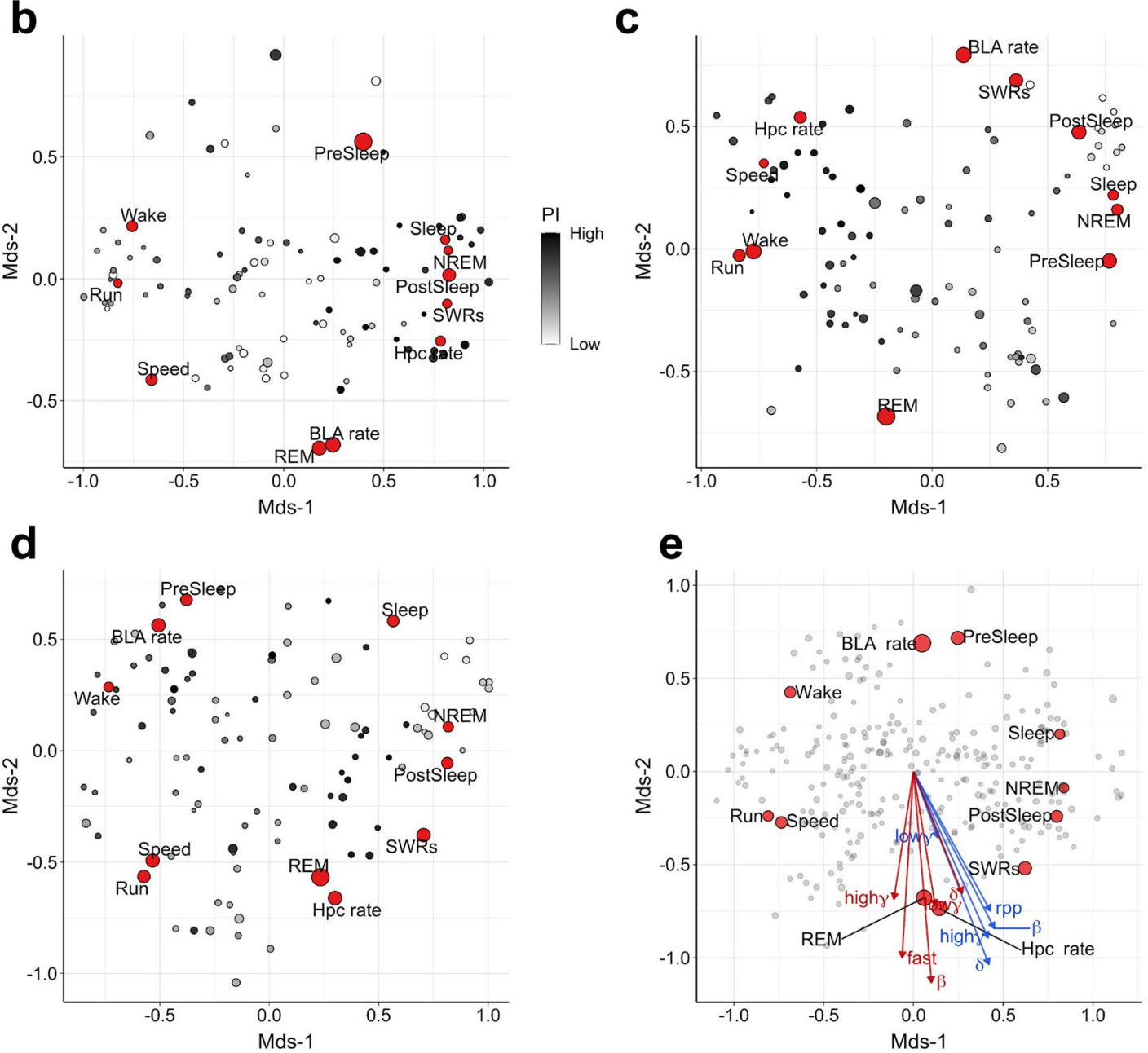

Figure 5. Unfolding projection of labels and clusters. $\boldsymbol{A}$, Each cluster (1) visualized by the SAPPHIRE plot and extracted by SbC (2) represents a particular combination of band powers (higher magnitude from green to red) and TC values (same palette of Fig. $2 A$ ). The 11 labels (or judges, see Materials and Methods, Unfolding projection), characterizing each time frame and displayed in the SAPPHIRE plot (2, orange box), are summarized in each cluster by their respective affinity scores (3). For each cluster, these values are computed either as fractions of occurrences for discrete labels, e.g., "PreSleep", "NREM", or as average values for continuous ones, e.g., "Hpc rate", "Speed". The affinity matrix is then used as a distance matrix to project both labels and clusters in a 2D space (4; see text for details). $\boldsymbol{B}-\boldsymbol{D}$, Unfolding projections for Rat1, Rat2, and Rat3, respectively. Clusters identified by the SbC method (gray-scale dots; brightness according to their mean PI values in the corresponding SAPPHIRE plots) and labels (red circles) are plotted in the reduced MDS space. The size of the dots is proportional to their contribution to the total stress. There are 89,89 , and 93 clusters for Rat1, Rat2, and Rat3, respectively. E, Unfolding projection for all three rats and biplot of the relevant mean TC components. Here, the color of the points is not related to any variable. The TCs were averaged over the leading bands but separately per direction of influence. The arrows show the results from fitting a plane in the three-dimensional space of MDS-1, MDS-2 and a 
continued

given mean TC. The direction gives the orientation of the plane, and the length is proportional to the slope. Blue arrows refer to hippocampus $\rightarrow$ BLA, red to the opposite. For the sake of visualization, Greek symbols and abbreviations of leading band names are used. Only planes with significant slopes are depicted in this way (permutation test: $n=271, p<0.05$ ). See details in Materials and Methods, Unfolding projection.

instead, which mismatches the various pairs of frequency ranges with respect to the band powers. In two rats and for both distributions, the true values indicate a significant role of TC coordination in discriminating the labels. For the remaining rat, coherently with Figure $6 A$, TC does not seem to provide improved performance. However, overall, we conjecture that the main contribution is provided by the LFP powers, which is reasonable given that many labels are directly correlated to it, e.g., NREM or REM phases or the presence of SWRs.

\section{Robustness of the SAPPHIRE methodology against alterna- tive preprocessing}

We tested the proposed analysis, which combines the SAPPHIRE plot with an unfolding method, also on an additional featurization with fewer dimensions (5) and alternative preprocessing (see Materials and Methods, Combined dataset of LFPs and TCs). Extended Data Figures 4-5, 4-6, 4-7 suggest that in this case the SAPPHIRE plots for the three rats are dominated by the band powers, which gives rise to a better separation of sleep phases and the appearance of a more detailed kinetic annotation. The strong clustering of "reward" labels in Extended Data Figure 4-7, which is not based on simple features like net BLA activity, is a striking example demonstrating that changes to the pipeline will be able to uncover further insights from these data in future work. There are two main reasons why we prefer the pipeline used for Figure 4 here. First, the original dimensionality (18) is such that TCs play a significant role in identifying clusters. Conversely, in a
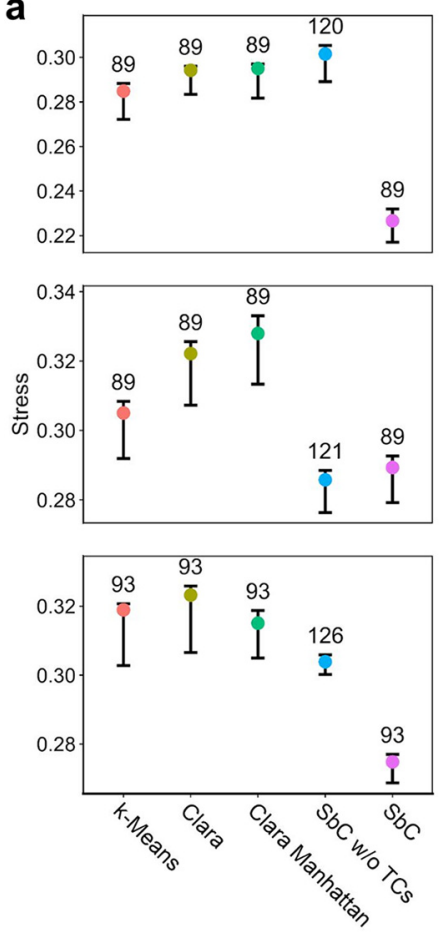

b
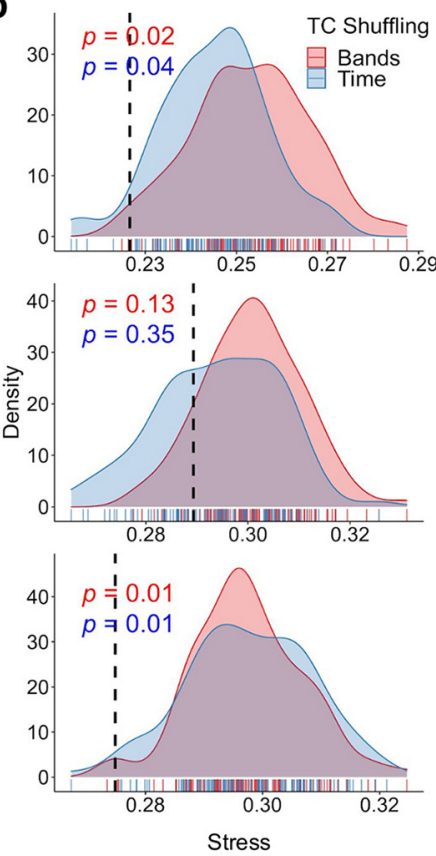

Figure 6. Comparison and analysis of the unfolding stress values. Top, middle, and bottom, Results for Rat1, Rat2, and Rat3, respectively. A, Stress values for SbC and clustering methods used commonly for large datasets. SbC results are included with and without inclusion of TCs. Error bars represent the $95 \%$ confidence interval resulting from jackknife resampling. The numbers of clusters are indicated on top. Note that a truly random embedding produces a distribution of much larger stress values, namely, $\sim 0.38$ for the 5th percentile (permutation of time bins after actual clustering). B, Distribution of stress values after application of the SbC clustering to two different randomized datasets. In blue, the 72 TC values were jointly shuffled in blocks along time within each session (randomizing only time while maintaining the coordination between TCs within each time block). The block size was equal to the average autocorrelation length across the 72 TCs. In red, for every time point, the 72 TC values themselves were permuted (randomizes only the pairs of power bands). For each type of shuffling, 100 permutations were performed. Vertical dashed lines indicate the actual values, and $p$ values are shown for each type of shuffling. The contribution of each unfolding label to the global stress value is investigated in Extended Data Figure 6-1 (see Materials and Methods). The analogous results derived from the combined dataset with alternative preprocessing are presented in Extended Data Figure 6-2. 
Extended Data Figures 4-5, 4-6, 4-7, TCs appear to not be strongly represented in the retained five principal components. Second, many of the smaller clusters suggested by the kinetic annotation have no apparent interpretation because of the coarseness of the available labels, which makes their interpretation challenging. From Extended Data Figure 6-2, it is clear that both preprocessing pipelines give similar ranges of stress scores, thus offering no reason to favor either. However, it is interesting to note that the results for the contribution of TC to the stress values are inverted across rats. Whereas the data for Rat2 fail to establish TC as substantial for reducing stress in Figure $6 B$, exactly the opposite is the case in Extended Data Figure 6-2F, where only Rat2 establishes TC as significant. Because we could not find hints in either behavior or TC values that could explain this dissimilarity with the other rats, we conjecture that the individuality of animals might require preprocessing pipelines to be tailored toward each animal, at least when the goal is to maximize sensitivity. Overall, however, two rather different pipelines (unweighted vs dynamically weighted features, dimensionality of 18 vs 5), produce SAPPHIRE plots that preserve, at a coarse level, both the mutual similarity of coarse states (like NREM sleep) and, approximately, the stress levels in the embedding. Thus, these data highlight the robustness of $\mathrm{SbC}$, in particular relative to the three approaches used for comparison.

\section{Discussion}

We propose here a set of methods for an unsupervised investigation of neural states and interaction patterns in complex datasets featuring recordings from multiple brain regions. We employed these methods to conduct a global and exploratory analysis of directed interactions between hippocampus and BLA, which relies on signal power alone. From the recorded LFPs, we extracted band powers in six different frequency bands and analyzed them in a three-stage approach. The dataset allowed us to conduct an extensive investigation of reciprocal influences between the two regions and to identify underlying neural states characterized by different levels and patterns of interactions across multiple epochs (Girardeau et al., 2017b). The methodological pipeline we presented above comprises unsupervised and scalable methods that take advantage of the entire recorded time series (see Materials and Methods, Progress index (PI) and SAPPHIRE plot). As such, our methodology is well-suited to a wide variety of complex neural data when the primary goal is an exploratory analysis of recurrent neural patterns. The three stages were: (1) a canonical comparison of interactions between regions across different behavioral and physiological time periods; (2) a high-resolution visualization procedure adopted from methods for the analysis of molecular dynamics simulations and (3) a global inference of putative neural states from a dataset combining time-resolved measures of reciprocal communication between the two brain regions with region-specific band powers. In the following discussion, we first examine benefits and limitations of the tools and workflow used during the analysis. We then focus on the biological insights that our analysis offers by recapitulating its salient aspects as well as discussing similarities and discrepancies with previous literature results.

\section{Methodological and theoretical insights \\ Directed interactions and the use of TC}

We chose TC (Harnack et al., 2017; Fig. 1C,D; Materials and Methods, Topological causality) to capture, at each time frame, potential bidirectional interactions and to quantify asymmetries in the strength of these reciprocal influences. In the presence of cycles or clear nonlinearities in the flow of information, it is probably more appropriate to analyze the system as a whole, that is, to view the time evolution of a variable $Y$ not merely as the sum of its present state and external inputs from $X$ but rather as the result of the joint history of the values of both $Y$ and $X$. This is related to the concept of non-separability of the system, which signifies that the history of $Y$ contains redundant information about $X$ that cannot be isolated and formally removed from the equations of motions of $Y$ (Sugihara et al., 2012). Thus, it is better to adopt here the notion of interdependence or "generalized synchrony/synchronization." This concept was established in multiple works in which the authors developed approaches to robustly quantify interdependence based on expansive mapping (Arnhold et al., 1999; Quiroga et al., 2000; for a review, see Pereda et al., 2005). These methods and TC have been applied to EEG data (see also Stam, 2005; Tajima et al., 2015, 2017), and their results encouraged the present application of TC to LFP recordings in the hippocampus-BLA system.

Many sophisticated methods provide valuable alternatives to evaluate nonlinear patterns of influence, such as nonlinear GC, dynamic causal modeling, and transfer entropy (Schreiber, 2000; Friston et al., 2003; Chen et al., 2004; Ishiguro et al., 2008), which, importantly, rely on different paradigms of functional connectivity estimation (Friston et al., 2013). However, we work with an actual biological dataset, and the absence of a ground truth makes it difficult to offer a sufficiently informative comparison across methods. Some comparative evaluations performed on test datasets can be found in the literature (see Gourévitch et al., 2006; Harnack et al., 2017; Krakovská et al., 2018).

The data-inferred interdependence and reciprocal influence between the two components of a nonlinear system differs from the event-driven cause and effect assessment performed by neural intervention and manipulation (Harnack et al., 2017; Jazayeri and Afraz, 2017). In the latter, a stimulus defined by the experimenter serves a causative role for (temporally) subsequent observations (the effect). This type of causation is typically deemed unequivocal as long as proper controls are in place. The main limitation is that most stimuli or interventions will only relate indirectly to the function of the brain. In contrast, a data-driven inference of directed interactions can in principle address and study functional units of the brain directly. In fact, these interactions could even be estimated accurately in a cause and effect sense if we were confident that our time series contain all the information necessary to fully describe the system's dynamics (Granger, 
1980). However, this is an unrealistic scenario both in general and for our analysis here given that we are able to observe only two brain regions at a time. If our data are not complete in the above sense, results have to be interpreted with caution. For example, some of the identified interactions may simply be the result of an external signal "passing through" in sequence. In other words, a driven interaction in the BLA-hippocampus system need not indicate an important role for either region in the process being observed. That said, TC does go beyond linear models of connectivity like GC and CCF, as, unlike those, it accounts for the non-separability of the system and can help elucidate details of the information exchange between hippocampus and amygdala (Sugihara et al., 2012; Harnack et al., 2017). The fact that some but not all of our results are congruent with prior results (Popa et al., 2010; Stujenske et al., 2014; Girardeau et al., 2017a), as detailed below, lends credibility to this mesoscopic, interactioncentric paradigm of analysis.

\section{On the use of spectrograms}

The use of multitaper spectrograms, the extraction of band powers from these, and the application of a quantile (rank) transformation on the time-resolved power values allowed us to compute TCs with globally consistent settings (see Materials and Methods, Topological causality). However, the adoption of this widespread filtering operation carries obvious downsides, in particular related to a potential loss of information and the blurring of interactions (de Cheveigné and Nelken, 2019). Specifically, as spectrograms average across a time window, here $2 \mathrm{~s}$, information on temporal details is lost, such as phases, or can be smoothed out in time, such as variations in amplitudes and details of SWRs. This blurring adds uncertainty to the localization in time of the interaction, and, generally speaking, can alter the apparent temporal precedence between two time series. However, as explained in Materials and Methods, Alignment settings, we tried to minimize the likelihood of such artifacts by deriving interactions from time series that we obtained with the same time-frequency analysis: the same spectrogram window was used for all the bands, and we maintained a consistent alignment between the resulting band powers throughout for deriving TCs (de Cheveigné and Nelken, 2019).

\section{On studying only power-power interactions}

The study of cross-frequency and high-frequency interactions largely involves phase-related measures, such as phase-amplitude and phase-phase couplings, as well as coherence (Lisman and Jensen, 2013; Fries, 2015). Whereas these analyses investigate fast timescales $(\sim 10 \mathrm{~ms})$, which are commonly interpreted to report on genuine physiological mechanisms, power-power or amplitude-amplitude interactions, which we study here, have been examined less often as their functional significance and mechanistic modalities are less clear (Canolty and Knight, 2010; Siegel et al., 2012). The higher timescales associated with these types of interactions $(\sim 0.1-1 \mathrm{~s})$ were observed in cross-frequency co-modulations between $\theta$ and $\gamma$ band powers in the hippocampus in Shirvalkar et al. (2010) and Buzsáki et al. (2003) where the authors employed similar spectrogram windows as those used here. In addition, it has been reported that trains of contiguous SWRs are detectable at these higher timescales (Fernández-Ruiz et al., 2019). Thus, the construction of spectrograms with its accompanying change in information content, helped by the nonlinear nature of TC, allows the inference that the interactions reported in this work might differ from and complement the ones found through a phasebased analysis. Clearly, our analysis is best-suited to identify mesoscopic interactions that are related to the generation of sustained rhythms, of sequences of burst events and, generally, of large irregular activity (Davidson et al., 2009; Palmigiano et al., 2017; van Ede et al., 2018; Fernández-Ruiz et al., 2019; Zich et al., 2020). This does not allow the inverse conclusion that other phenomena cannot be observed at all, however. As Figure $1 E$ shows, the resultant TCs do in fact vary quickly in time, which is further enhanced by the fact that we zero non-significant values in the TC time series (see Materials and Methods, Topological causality). In summary, our analysis goes beyond the (large) part of the literature that deals with (filtered) signals at higher resolution and focuses on coherent oscillations.

Advantages of the proposed methodology for the identification of neural states

We combined the information provided by the band powers and TCs to search for a possible temporal recurrence of interaction patterns. To this end, we concatenated data from multiple recording sessions for each of the rats, thus considering a large dataset $\left(\sim 10^{6}\right.$ time frames). Importantly, we did not apply any pre-selection of temporal windows to be analyzed by, e.g., restricting ourselves to sleep phases, SWRs frames, or periods with high firing rates. Moreover, the assessment of clustering performance employed a set of available labels commonly adopted to characterize individual frames in these time series. The $\mathrm{SbC}$ method performs better than three commonly employed clustering tools for all the animals analyzed, and this remains valid even if the data are preprocessed differently (Extended Data Fig. 6-2). We hypothesize that the main advantage with respect to other techniques consists in using explicitly the temporal information. This is done by evaluating both the recurrence of the states along the time series and the actual kinetic distance between them. Importantly, we showed that TC provides in most of the cases a significant contribution in discriminating the different behavioral and neural labels that annotate the recordings. Generally, there are no particular limitations in extending application of the SAPPHIRE plots and subsequent analyses to other types of neural dataset with different experimental settings (see also Garolini et al., 2019). For example, in a typical working memory experiment, the animal repeatedly performs a task with fixed temporal structure under different contexts. The SAPPHIRE plot resolves this single-trial structure and may thus be used to investigate whether any of the trial epochs allow a better distinction of one or more of the different contexts.

\section{Biological insights}

Physiological connection of hippocampus and BLA and biological limitations of the analysis

The ventral part of the hippocampus projects directly to the BLA, in contrast to the dorsal one, which is what is 
studied in the original dataset and analyzed here. However, as remarked in Girardeau et al. (2017a), the interactions between the dorsal hippocampus and the BLA could be mediated by indirect pathways, with particular attention given to the entorhinal cortex (Bauer et al., 2007; Colgin, 2016). In this regard, it is also important to note that our analysis relies only on the LFP signals, across all bands, and does not use spike information. Coordination between LFPs in the dorsal hippocampus and the BLA has in fact been observed in the $\theta$ (Seidenbecher et al., 2003; Bienvenu et al., 2012) and high- $\gamma$ (Stujenske et al., 2014) bands, and the results of Girardeau and colleagues highlight the importance of SWRs for such coordinated activity. Importantly, the nature of the data and the interactions we are able to analyze address purely the issue of functional connectivity between the two regions rather than the issue of direct projections.

There are further considerations that might limit the biological interpretation of the results obtained as follows. In our analysis, band powers or other features of the LFP signal have not been thresholded to single out selected oscillatory events. Given this, the known $1 / f$ trend in the LFP spectrum in a broad and heterogenous state such as NREM should be accounted for. Similarly, it has been observed that $\beta$ and part of the low- $\gamma$ oscillations in the hippocampus arise from overlapping effects of SWRs during NREM sleep (Oliva et al., 2018). Finally, volume conduction might interfere with signals in low-frequency bands (Łesski et al., 2013). Specifically for the $\delta$ and $\theta$ bands, we tried to estimate its role in the Results section (see Interaction analysis between behavioral epochs and sleep phases) and in Extended Data Figure 2-2: while we can reasonably deduce that the contribution is marginal for $\theta$, we did not arrive at the same conclusion for $\delta$. In particular, oscillations traveling from the hippocampus to the BLA via volume conduction (Bertone-Cueto et al., 2020) pose the scenario that BLA $\delta$-driven interactions detected via TC are actually indicative of activity and interactions within the hippocampus alone. All of these qualifying considerations should be kept in mind when interpreting some of the details of our results.

\section{Interaction patterns in REM and NREM sleep}

One of our main observations is that reciprocal influences between hippocampus and BLA are particularly sustained during NREM sleep and that this mutual influence is carried predominantly by the low-frequency bands, namely $\delta, \theta$, and $\beta$. During REM sleep, a general reduction of interactions is observed in these bands and interareal communication appears to be mediated by the respective fast frequencies. The notable exception to this are hippocampus-driven TCs in the $\theta$ band, which are actually stronger than in NREM sleep. We conjecture that the more complex interplay of different frequencies, along with the shorter duration and lower regularity of activity in REM sleep, is the likely culprit for the apparent lack of correlation between the various interaction measures in Figure $2 B, C$, bottom panels. While the slightly larger differences between pre-sleep and post-sleep appearing in NREM with respect to REM sleep (Figs. 2A, 3) appear to corroborate the findings of the original analysis of the same data (Girardeau et al., 2017b), our results for REM sleep are at odds with the role REM sleep is commonly assigned to play in consolidating emotional memories (Genzel et al., 2015; Hutchison and Rathore, 2015; Boyce et al., 2016). For example, the work of Popa et al. (2010) demonstrated that an increase in the $\theta$ coherence during REM between hippocampus, BLA, and medial prefrontal cortex (mPFC) correlated with the consolidation of conditioned fear. In agreement with their Granger analysis, we do observe an apparent, mostly one-sided influence of the hippocampal $\theta$ on the BLA $\theta$ band (Fig. $2 A$ ). If we extend the view to other frequency bands, we find that the BLA $\theta$ influence on various hippocampal bands decreases significantly with respect to NREM sleep whereas the hippocampal $\theta$ band is significantly enhanced also in its influence on higher BLA frequencies. However, and this differs from expectation, we do not observe any significant contribution of the $\theta$ band, in either region, to mark differences between pre-sleep and post-sleep REM epochs (Fig. 2A). This is despite the fact that the BLA $\theta$-driven TCs are one of the channels that appears to be able to distinguish pre-run from post-run in the aversive direction (Extended Data Fig. 2-1).

In another work (Stujenske et al., 2014), high- $\gamma$ BLA activity during wakefulness was observed to drive ventral hippocampal high frequencies during safety signals. In contrast, we do not observe a specific pattern for the safe direction (Extended Data Fig. 2-1), which is probably expected given the established differences in the roles of the ventral and dorsal regions of the hippocampus (Fanselow and Dong, 2010). During NREM sleep, reciprocal interactions between the two lowest frequency ranges may originate instead from the sequential processing in hippocampus and BLA of slow oscillatory signals originating from the cortex (Genzel et al., 2014, 2015). Interestingly, many of the significant differences between post-sleep and pre-sleep episodes or between aversive and safe directions concern communication from the BLA to the hippocampus with the $\beta$ band of the amygdala playing a prominent role (Fig. 3; Extended Data Fig. 3-2). To our knowledge, $\beta$ oscillation bursts in the amygdala have been characterized in detail only in Stujenske et al. (2014) where they were triggered by an auditory cue preceding an aversive stimulus. Differently from that work, the data we analyzed are from experiments where rats perform a nonPavlovian spatial task. It is thus difficult to provide an exact side-by-side comparison of our results for the $\beta$ band to those of Stujenske et al. (2014).

\section{The role of SWRs}

We provide additional evidence that SWRs are a hallmark of episodes in which the hippocampus exerts a directed influence on the BLA whether during sleep or not (Fig. 3; Extended Data Fig. 3-2). Interestingly, the TC analysis indicates that this influence is not confined to the ripples band but also mediated through the $\delta, \theta$, and $\beta$ bands. SWRs play a prominent role in memory consolidation and retrieval, since they seem to provide a mechanism for facilitating the transfer of hippocampal memories to connected brain regions (Girardeau and Zugaro, 2011; Buzsáki, 2015; Joo and Frank, 2018; Laventure and Benchenane, 2020). As shown in Girardeau and colleagues, hippocampal SWRs upmodulate precisely those 
BLA cells that contribute the most to the joint neural reactivations in post-NREM sleep. In our analysis, while SWRs are a significant indicator of directed interactions with the BLA driven by the hippocampus (Fig. 3), there are barely any significant differences in the interaction patterns, neither when considering the entire pre/post-sleep epochs (Fig. 2A) nor when restricting the analysis to NREM sleep (Fig. 3). To us, the most likely scenario explaining this lack of contrast is threefold. First, the experiment employs ubiquitous positive stimuli in the form of water rewards. They are present whenever the rat is on the track (Fig. 1A) and might provide a strong background signal of reciprocal communication. Second, the only clear evidence we see of contrast between pre/post-epochs is for the pre/ post-run phases (Extended Data Fig. 2-1), which differ only in that the negative stimuli were encountered more recently (time scale of 2-3 h) in the post-run phase. This suggests that the timing of memory retrieval/processing is specific. Third, our power band-based analysis might lack the necessary sensitivity, in particular in the ripples band.

\section{A functional view of neural activity in the form of well-de- fined states}

The time series analyzed, i.e., LFP power band contributions and the TCs derived from them, belong to a mesoscopic level of observation. This representation of the neuronal state of the joint BLA-hippocampus system provided us with a coarse characterization of the system. For example, in Figure 4 (Rat3), we identify, in an unsupervised manner, four coarse states (from left to right): NREM sleep with low interactions but high SWR activity; activity involving emotional processing (both reward and fear) with directed interactions but low SWRs; a waking but seemingly inactive state with neither interactions nor SWRs; finally, a mixed state of NREM sleep and activity with emotional processing where both the reciprocal influence and SWRs are high. The (few) REM time points are embedded in the first active state (second from the left), and, to a lesser extent, also in the last mixed state, which is consistent with the well-known similarities between neural activities in REM and wake phases. The last (rightmost) state is the most interesting one given that it features mostly SWRs, NREM sleep, and both aversive and reward events while carrying a signature of strong interactions. This latter state is a potential candidate for addressing the joint neuronal reactivations that capture the emotional and contextual components of the run epoch (Girardeau et al., 2017a). However, since we could not find a clear distinction between pre-sleep and post-sleep epochs in our unsupervised analysis, the data suggest either that the discriminating patterns are too sporadic to create well-defined states, or that information about spiking activity is of critical importance and must be accounted for in the dataset.

In the spirit of an exploratory analysis, we did not formulate a specific hypothesis in this work. We rather adopted a set of computational tools, some of which were originally developed for completely different tasks, for an unbiased, data-driven exploration of mutual interactions between amygdala and hippocampus across different types of neural activities. Because we use band powers rather than raw LFPs to infer directed interactions between these two brain regions, our analysis is focused on function at mesoscopic resolution rather than on anatomic connections at the level of specific groups of neurons. The ultimate scope of the results presented here is to suggest new hypotheses to be tested by confirmatory research (Schwab and Held, 2020). For example, we hypothesize a role for the BLA $\beta$ band in promoting memory consolidation (Fig. 3), which is putatively associated with the processing of negative stimuli (Extended Data Fig. 32). Similarly, based on the third coarse state in Figure 4, we conjectured that there is a neurologically homogeneous "resting" state in which amygdala and hippocampus are, except for a striking absence of SWRs, active but decoupled. We hope that our work will help motivate future analyses and experiments aimed at testing hypotheses like the above.

\section{References}

Aggarwal CC, Hinneburg A, Keim DA (2001) On the surprising behavior of distance metrics in high dimensional space. In: Database theory - ICDT 2001, lecture notes in computer science, pp 420434. Berlin; Heidelberg: Springer.

Arnhold J, Grassberger P, Lehnertz K, Elger CE (1999) A robust method for detecting interdependences: application to intracranially recorded EEG. Physica D 134:419-430.

Babadi B, Brown EN (2014) A review of multitaper spectral analysis. IEEE Trans Biomed Eng 61:1555-1564.

Barnett L, Seth AK (2014) The MVGC multivariate granger causality toolbox: a new approach to granger-causal inference. J Neurosci Methods 223:50-68.

Bauer EP, Paz R, Paré D (2007) Gamma oscillations coordinate amygdalo-rhinal interactions during learning. J Neurosci 27:93699379.

Behrisch M, Bach B, Henry Riche N, Schreck T, Fekete J-D (2016) Matrix reordering methods for table and network visualization. Comput Graph Forum 35:693-716.

Benjamini Y, Yekutieli D (2001) The control of the false discovery rate in multiple testing under dependency. Ann Stat 29:1165-1188.

Bertone-Cueto NI, Makarova J, Mosqueira A, García-Violini D, Sánchez-Peña R, Herreras O, Belluscio M, Piriz J (2020) Volumeconducted origin of the field potential at the lateral habenula. Front Syst Neurosci 13:78.

Beyeler A, Namburi P, Glober GF, Simonnet C, Calhoon GG, Conyers GF, Luck R, Wildes CP, Tye KM (2016) Divergent routing of positive and negative information from the amygdala during memory retrieval. Neuron 90:348-361.

Bienvenu TCM, Busti D, Magill PJ, Ferraguti F, Capogna M (2012) Cell-type-specific recruitment of amygdala interneurons to hippocampal theta rhythm and noxious stimuli in vivo. Neuron 74:10591074.

Blöchliger N, Vitalis A, Caflisch A (2013) A scalable algorithm to order and annotate continuous observations reveals the metastable states visited by dynamical systems. Comput Phys Commun 184:2446-2453.

Blöchliger N, Vitalis A, Caflisch A (2014) High-resolution visualisation of the states and pathways sampled in molecular dynamics simulations. Sci Rep 4:6264.

Blöchliger N, Caflisch A, Vitalis A (2015) Weighted distance functions improve analysis of high-dimensional data: application to molecular dynamics simulations. J Chem Theory Comput 11:5481-5492.

Bocchio M, Nabavi S, Capogna M (2017) Synaptic plasticity, engrams, and network oscillations in amygdala circuits for storage and retrieval of emotional memories. Neuron 94:731-743. 
Borg I, Groenen PJF, Mair P (2018) Applied multidimensional scaling and unfolding. Berlin: Springer International Publishing.

Boyce R, Glasgow SD, Williams S, Adamantidis A (2016) Causal evidence for the role of REM sleep theta rhythm in contextual memory consolidation. Science 352:812-816.

Bradley E, Kantz H (2015) Nonlinear time-series analysis revisited. Chaos 25:097610.

Buzsáki G (2015) Hippocampal sharp wave-ripple: a cognitive biomarker for episodic memory and planning. Hippocampus 25:1073-1188.

Buzsáki G, Draguhn A (2004) Neuronal oscillations in cortical networks. Science 304:1926-1929.

Buzsáki G, Buhl DL, Harris KD, Csicsvari J, Czéh B, Morozov A (2003) Hippocampal network patterns of activity in the mouse. Neuroscience 116:201-211.

Canolty RT, Knight RT (2010) The functional role of cross-frequency coupling. Trends Cogn Sci 14:506-515.

Cao L, Mees A, Judd K (1998) Dynamics from multivariate time series. Physica D 121:75-88.

Chen SX (1999) Beta kernel estimators for density functions. Comput Stat Data Anal 31:131-145.

Chen Y, Rangarajan G, Feng J, Ding M (2004) Analyzing multiple nonlinear time series with extended granger causality. Phys Lett $A$ 324:26-35.

Chernick MR, LaBudde RA (2014) An introduction to bootstrap methods with applications to R. Hoboken: Wiley.

Cocina F, Vitalis A, Caflisch A (2020) SAPPHIRE-based clustering. J Chem Theory Comput 16:6383-6396.

Colgin LL (2016) Rhythms of the hippocampal network. Nat Rev Neurosci 17:239-249.

Cox TF, Cox MAA (2000) Multidimensional scaling, Ed 2. Milton Park: Taylor and Francis.

Davidson TJ, Kloosterman F, Wilson MA (2009) Hippocampal replay of extended experience. Neuron 63:497-507.

de Cheveigné A, Nelken I (2019) Filters: when, why, and how (not) to use them. Neuron 102:280-293.

de Leeuw J, Mair P (2009) Multidimensional scaling using majorization: SMACOF in R. J Stat Softw 31:1-30.

Efron B, Tibshirani RJ (1994) An introduction to the bootstrap. Boca Raton: CRC

Fanselow MS, Dong H-W (2010) Are the dorsal and ventral hippocampus functionally distinct structures? Neuron 65:7-19.

Fernández-Ruiz A, Oliva A, de Oliveira EF, Rocha-Almeida F, Tingley D, Buzsáki G (2019) Long-duration hippocampal sharp wave ripples improve memory. Science 364:1082-1086.

Fraser AM, Swinney HL (1986) Independent coordinates for strange attractors from mutual information. Phys Rev A 33:1134-1140.

Fries $P$ (2015) Rhythms for cognition: communication through coherence. Neuron 88:220-235.

Friston K, Moran R, Seth AK (2013) Analysing connectivity with granger causality and dynamic causal modelling. Curr Opin Neurobiol 23:172-178.

Friston KJ, Harrison L, Penny W (2003) Dynamic causal modelling. Neuroimage 19:1273-1302.

Garolini D, Vitalis A, Caflisch A (2019) Unsupervised identification of states from voltage recordings of neural networks. J Neurosci Methods 318:104-117.

Genzel L, Kroes MCW, Dresler M, Battaglia FP (2014) Light sleep versus slow wave sleep in memory consolidation: a question of global versus local processes? Trends Neurosci 37:10-19.

Genzel L, Spoormaker VI, Konrad BN, Dresler M (2015) The role of rapid eye movement sleep for amygdala-related memory processing. Neurobiol Learn Mem 122:110-121.

Girardeau G, Zugaro M (2011) Hippocampal ripples and memory consolidation. Curr Opin Neurobiol 21:452-459.

Girardeau G, Inema I, Buzsáki G (2017a) Reactivations of emotional memory in the hippocampus-amygdala system during sleep. Nat Neurosci 20:1634-1642.

Girardeau G, Inema I, Buzsaki G (2017b) Simultaneous large-scale recordings in dorsal hippocampus, basolateral amygdala and neighbouring deep nuclei and structures in rats performing a spatial aversive task and sleeping. CRCNS. Available at http://dx.doi. org/10.6080/KOMS3QXD.

Gourévitch B, Bouquin-Jeannès RL, Faucon G (2006) Linear and nonlinear causality between signals: methods, examples and neurophysiological applications. Biol Cybern 95:349-369.

Granger CWJ (1980) Testing for causality: a personal viewpoint. J Econ Dyn Control 2:329-352.

Gründemann J, Bitterman Y, Lu T, Krabbe S, Grewe BF, Schnitzer MJ, Lüthi A (2019) Amygdala ensembles encode behavioral states. Science 364:

Hahsler M, Hornik K, Buchta C (2008) Getting things in order: an introduction to the R package seriation. J Stat Softw 25:1-34.

Harnack D, Laminski E, Schünemann M, Pawelzik KR (2017) Topological causality in dynamical systems. Phys Rev Lett 119:098301.

Hegger R, Kantz H, Matassini L, Schreiber T (2000) Coping with nonstationarity by overembedding. Phys Rev Lett 84:4092-4095.

Hutchison IC, Rathore S (2015) The role of REM sleep theta activity in emotional memory. Front Psychol 6:1439.

Ishiguro K, Otsu N, Lungarella M, Kuniyoshi Y (2008) Comparison of nonlinear granger causality extensions for low-dimensional systems. Phys Rev E Stat Nonlin Soft Matter Phys 77:036217.

Jazayeri M, Afraz A (2017) Navigating the neural space in search of the neural code. Neuron 93:1003-1014.

Joo HR, Frank LM (2018) The hippocampal sharp wave-ripple in memory retrieval for immediate use and consolidation. Nat Rev Neurosci 19:744-757.

Kantz H, Schreiber T (2004) Nonlinear time series analysis. Cambridge: Cambridge University Press.

Kim SY, Payne JD (2020) Neural correlates of sleep, stress, and selective memory consolidation. Curr Opin Behav Sci 33:57-64.

Kirkby LA, Luongo FJ, Lee MB, Nahum M, Van Vleet TM, Rao VR, Dawes HE, Chang EF, Sohal VS (2018) An amygdala-hippocampus subnetwork that encodes variation in human mood. Cell 175:1688-1700.e14.

Klein M, Krivov SV, Ferrer AJ, Luo L, Samuel AD, Karplus M (2017) Exploratory search during directed navigation in $\mathrm{C}$. elegans and Drosophila larva. eLife 6:e30503.

Krakovská A, Jakubík J, Chvosteková M, Coufal D, Jajcay N, Paluš M (2018) Comparison of six methods for the detection of causality in a bivariate time series. Phys Rev E 97:e042207.

Kyriazi P, Headley DB, Pare D (2018) Multi-dimensional coding by basolateral amygdala neurons. Neuron 99:1315-1328.e5.

Laventure S, Benchenane K (2020) Validating the theoretical bases of sleep reactivation during sharp-wave ripples and their association with emotional valence. Hippocampus 30:19-27.

Łesski S, Lindén H, Tetzlaff T, Pettersen KH, Einevoll GT (2013) Frequency dependence of signal power and spatial reach of the local field potential. PLoS Comput Biol 9:e1003137.

Likhtik E, Stujenske JM, Topiwala MA, Harris AZ, Gordon JA (2014) Prefrontal entrainment of amygdala activity signals safety in learned fear and innate anxiety. Nat Neurosci 17:106-113.

Lisman JE, Jensen O (2013) The $\vartheta-\gamma$ neural code. Neuron 77:10021016.

Maingret N, Girardeau G, Todorova R, Goutierre M, Zugaro M (2016) Hippocampo-cortical coupling mediates memory consolidation during sleep. Nat Neurosci 19:959-964.

Narayanan RT, Seidenbecher T, Kluge C, Bergado J, Stork O, Pape HC (2007) Dissociated theta phase synchronization in amygdalohippocampal circuits during various stages of fear memory. Eur $\mathrm{J}$ Neurosci 25:1823-1831.

Nolte G, Bai O, Wheaton L, Mari Z, Vorbach S, Hallett M (2004) Identifying true brain interaction from EEG data using the imaginary part of coherency. Clin Neurophysiol 115:2292-2307.

Oliva A, Fernández-Ruiz A, Fermino de Oliveira E, Buzsáki G (2018) Origin of gamma frequency power during hippocampal sharpwave ripples. Cell Rep 25:1693-1700.e4.

Palmigiano A, Geisel T, Wolf F, Battaglia D (2017) Flexible information routing by transient synchrony. Nat Neurosci 20:1014-1022. 
Pape HC, Pare D (2010) Plastic synaptic networks of the amygdala for the acquisition, expression, and extinction of conditioned fear. Physiol Rev 90:419-463.

Paré D, Collins DR, Pelletier JG (2002) Amygdala oscillations and the consolidation of emotional memories. Trends Cogn Sci 6:306314.

Pereda E, Quiroga RQ, Bhattacharya J (2005) Nonlinear multivariate analysis of neurophysiological signals. Prog Neurobiol 77:1-37.

Pesaran B, Vinck M, Einevoll GT, Sirota A, Fries P, Siegel M, Truccolo W, Schroeder CE, Srinivasan R (2018) Investigating large-scale brain dynamics using field potential recordings: analysis and interpretation. Nat Neurosci 21:903-919.

Phillips RG, LeDoux JE (1992) Differential contribution of amygdala and hippocampus to cued and contextual fear conditioning. Behav Neurosci 106:274-285.

Popa D, Duvarci S, Popescu AT, Léna C, Paré D (2010) Coherent amygdalocortical theta promotes fear memory consolidation during paradoxical sleep. Proc Natl Acad Sci USA 107:6516-6519.

Purpura KP, Bokil HS (2008) Neural signal processing: tutorial I. In: Neural signal processing: quantitative analysis of neural activity (Mitra P, ed), pp 67-77. Washington, DC: Society for Neuroscience.

Quiroga RQ, Arnhold J, Grassberger P (2000) Learning driver-response relationships from synchronization patterns. Phys Rev E 61:5142-5148. Pt A):

Sawangjit A, Oyanedel CN, Niethard N, Salazar C, Born J, Inostroza $M(2018)$ The hippocampus is crucial for forming non-hippocampal long-term memory during sleep. Nature 564:109-113.

Schapiro AC, Reid AG, Morgan A, Manoach DS, Verfaellie M, Stickgold R (2019) The hippocampus is necessary for the consolidation of a task that does not require the hippocampus for initial learning. Hippocampus 29:1091-1100.

Schreiber T (2000) Measuring information transfer. Phys Rev Lett 85:461-464.

Schwab S, Held L (2020) Different worlds confirmatory versus exploratory research. Significance 17:8-9.

Seidenbecher T, Laxmi TR, Stork O, Pape H-C (2003) Amygdalar and hippocampal theta rhythm synchronization during fear memory retrieval. Science 301:846-850.

Sheikhattar A, Miran S, Liu J, Fritz JB, Shamma SA, Kanold PO, Babadi B (2018) Extracting neuronal functional network dynamics via adaptive granger causality analysis. Proc Natl Acad Sci USA 115:E3869-E3878.

Shinomoto S, Kim H, Shimokawa T, Matsuno N, Funahashi S, Shima K, Fujita I, Tamura H, Doi T, Kawano K, Inaba N, Fukushima K, Kurkin S, Kurata K, Taira M, Tsutsui KI, Komatsu H, Ogawa T, Koida K, Tanji J, et al. (2009) Relating neuronal firing patterns to functional differentiation of cerebral cortex. PLoS Comput Biol 5: e1000433.
Shirvalkar PR, Rapp PR, Shapiro ML (2010) Bidirectional changes to hippocampal theta-gamma comodulation predict memory for recent spatial episodes. Proc Natl Acad Sci USA 107:7054-7059.

Siegel M, Donner TH, Engel AK (2012) Spectral fingerprints of largescale neuronal interactions. Nat Rev Neurosci 13:121-134.

Stam CJ (2005) Nonlinear dynamical analysis of EEG and MEG: review of an emerging field. Clin Neurophysiol 116:2266-2301.

Stujenske JM, Likhtik E, Topiwala MA, Gordon JA (2014) Fear and safety engage competing patterns of theta-gamma coupling in the basolateral amygdala. Neuron 83:919-933.

Sugihara G, May RM (1990) Nonlinear forecasting as a way of distinguishing chaos from measurement error in time series. Nature 344:734-741.

Sugihara G, May R, Ye H, Hsieh C-H, Deyle E, Fogarty M, Munch S (2012) Detecting causality in complex ecosystems. Science 338:496-500.

Tajima S, Yanagawa T, Fujii N, Toyoizumi T (2015) Untangling brainwide dynamics in consciousness by cross-embedding. PLoS Comput Biol 11:e1004537.

Tajima S, Mita T, Bakkum DJ, Takahashi H, Toyoizumi T (2017) Locally embedded presages of global network bursts. Proc Natl Acad Sci USA 114:9517-9522.

Takens F (1981) Detecting strange attractors in turbulence. In: Dynamical systems and turbulence, Warwick 1980. pp 366-381. Berlin; Heidelberg: Springer.

van Ede F, Quinn AJ, Woolrich MW, Nobre AC (2018) Neural oscillations: sustained rhythms or transient burst-events? Trends Neurosci 41:415-417.

Vitalis A, Caflisch A (2012) Efficient construction of mesostate networks from molecular dynamics trajectories. J Chem Theory 8:1108-1120.

Zelikowsky M, Hersman S, Chawla MK, Barnes CA, Fanselow MS (2014) Neuronal ensembles in amygdala, hippocampus, and prefrontal cortex track differential components of contextual fear. $\mathrm{J}$ Neurosci 34:8462-8466.

Zhang T, Ramakrishnan R, Livny M (1996) BIRCH: an efficient data clustering method for very large databases. SIGMOD Rec 25:103114.

Zheng J, Anderson KL, Leal SL, Shestyuk A, Gulsen G, Mnatsakanyan L, Vadera S, Hsu FPK, Yassa MA, Knight RT, Lin JJ (2017) Amygdala-hippocampal dynamics during salient information processing. Nat Commun 8:14413.

Zheng J, Stevenson RF, Mander BA, Mnatsakanyan L, Hsu FPK, Vadera S, Knight RT, Yassa MA, Lin JJ (2019) Multiplexing of theta and alpha rhythms in the amygdala-hippocampal circuit supports pattern separation of emotional information. Neuron 102:887-898. e5.

Zich C, Quinn AJ, Mardell LC, Ward NS, Bestmann S (2020) Dissecting transient burst events. Trends Cogn Sci 24:784-788. 